Extrême-Orient Extrême-Occident

\section{Extrême-Orient Extrême-Occident}

38 | 2014

La Guerre en perspective : histoire et culture militaire en Chine

Écrire l'histoire vis-à-vis de la guerre : postures historiennes, conceptions et récits de l'histoire sous la République de Chine (1912-1949)

Historical Writing in Times of War: Historians, Conceptions and Narratives of

History in Republican China

戰時歷史書寫: 中國民國時期的史學家及歷史觀念和敘事

\title{
Damien Morier-Genoud
}

\section{OpenEdition}

Journals

Édition électronique

URL : http://journals.openedition.org/extremeorient/377

DOI : $10.4000 /$ extremeorient.377

ISSN : 2108-7105

Éditeur

Presses universitaires de Vincennes

Édition imprimée

Date de publication : 1 novembre 2014

Pagination : 170-206

ISBN : 978-2-84292-414-0

ISSN : 0754-5010

Référence électronique

Damien Morier-Genoud, «Écrire l'histoire vis-à-vis de la guerre : postures historiennes, conceptions et récits de l'histoire sous la République de Chine (1912-1949) ", Extrême-Orient Extrême-Occident [En ligne], 38 | 2014, mis en ligne le 01 janvier 2017, consulté le 19 avril 2019. URL : http:// journals.openedition.org/extremeorient/377 ; DOI : 10.4000/extremeorient.377 


\title{
Écrire l'histoire vis-à-vis de la guerre: postures historiennes, conceptions et récits de l'histoire sous la République de Chine (1912-1949)
}

\author{
Damien Morier-Genoud
}

«Comme les vagues de l'océan qui renaissent de leur échec même, c'est leur inconsistance qui les rend indestructibles. »C'est par cette belle image aux exhalaisons d'écume et de sel, et avec l'indignation qui le caractérise, que Simon Leys fustigeait ces soi-disant «experts» qui, une fois leurs salons parisiens retrouvés, expliquaient à leur petit monde les monts et merveilles d'une Chine maoïste dont ils n'avaient arpenté que les recoins les plus lisses ${ }^{1}$. Les recoins les plus lisses ou les seules réalités édulcorées que le régime leur avait laissé voir. À goûter de plus près l'analogie maritime de notre sinologue, on est tenté de suggérer qu'elle pourrait presque tout aussi bien s'appliquer aux guerres qui n'ont eu de cesse de ravager l'humanité dans l'histoire. Car c'est bien de la défaite des guerres que renaissent les guerres, celles du moment, aussi identifiables qu'identiques. Alors toujours faut-il qu'après les souffrances et les blessures, les atrocités, l'horreur et la mort, rejaillisse le bruit des armes, qu'on blâme toujours et encore. Et chacun à nouveau de pleurer ses défunts, d'enterrer le passé, de prendre acte, d'admettre enfin que la seule victoire qu'une guerre pourra à jamais remporter sera celle de ne jamais recommencer. Si toutes les guerres, du reste, diffèrent dans l'âpre moment de leur surrection autant qu'elles convergent dans une opiniâtre récurrence, quelle attitude adoptent les historiens vis-à-vis d'elles? Et que révèlent les conceptions et pratiques de l'histoire dans la Chine républicaine - une Chine marquée par son lot de violence et de chaos - de cette posture historienne face au champ de bataille? Telle sera la question de départ du présent article.

Guerre et histoire, donc. Le rapprochement s'y prête tant celle-ci entre d'emblée en résonance avec celle-là. Qu'elle soit prise comme totalité de l'expérience humaine amoncelée, comme discipline du savoir ou suite d'événements racontés, l'histoire ne se donne-t-elle pas à entendre comme un

1. Leys (1998): 705-714. 
champ de bataille perpétuel? Ni les cessez-le-feu, ni le clairon des grandes théories et des grands récits ne semblent du moins avoir été jusque là en mesure de faire taire la sourde rumeur des guerres qui déchire et sous-tend l'histoire de part en part. Cet enchevêtrement intime de la guerre et de l'histoire ne date pas d'hier, loin s'en faut. C'est en sondant la première que, dès l'antiquité grecque, Thucydide redéfinissait la seconde comme quête de la vérité et la faisait ainsi sortir du mythe ${ }^{2}$. Plus proche de notre contemporain, Schiller évoque lui aussi le caractère indissociable de l'une et l'autre quand, dans une conférence de 1789 , il soutient que l'Histoire «abaisse un regard également serein sur les travaux sanglants de la guerre et sur les peuples paisibles qui se nourrissent innocemment du lait de leurs troupeaux ${ }^{3}$. Certes le poète allemand suggère ici que le temps de la guerre alterne avec celui de la paix. Mais dans certaines contrées où les conflits armés ne laissent de renaître de leurs cendres, tout porte à croire que les armistices ne tiennent lieu que de chimères. Ils ne valent que pour leur propension à amplifier de façon toujours plus criante les déclarations de guerre qui leur succèdent inexorablement. Dans ces contrées-là - l'Irak du $\mathrm{XX}^{\mathrm{e}}-\mathrm{XXI}^{\mathrm{e}}$ siècle en est à lui seul un bon exemple -, la paix apparaît telle une respiration qui n'altère en rien le tempo effréné de la partition de la guerre. Elle est, pour ainsi dire, la partie en creux, l'intaille par défaut du camée de la guerre. Et quand l' «Histoire», témoin de l'action des hommes, regarde dans l'indifférence les conflits et les trêves, l' «histoire», produit de l'action des hommes, se confond tout entière avec eux.

Sous l'angle de son écriture et des possibles interprétations dont elle fait l'objet, l'histoire, nous l'avons dit, est aussi le lieu de tous les affrontements. C'est ce qu'annonce ainsi sans détour, dès sa première de couverture, un ouvrage d'Enzo Traverso ${ }^{4}$. D'où la multiplication ces vingt dernières années d'histoires qui se proposent de retracer les évolutions mêmes de la discipline historique. Cette tendance, conjointement représentée, du côté français, par des historiens comme Christian Delacroix, François Dosse et Patrick Garcia ${ }^{5}$, témoigne d'un souci réflexif comme d'un besoin impérieux de la discipline: scruter les expériences historiques et culturelles - précisément antagonistes et belliqueuses - dont elle procède elle-même en tant qu'objet.

Guerre dans l'histoire et guerre de l'histoire... À la guerre létale que se livrent entre eux les hommes par les armes, avec sa palette d'arts, de stratégies et de moyens allant de la poliorcétique au «chirurgical», en passant par les

2. Sur cette question, voir Romilly (2005).

3. Schiller (1872): 422.

4. Traverso (2011).

5. Voir Delacroix, Dosse et Garcia (2007). 
tranchées et la blitzkrieg, vient s'accoler la guerre des mots, des idées et, plus récemment, des mémoires. C'est d'ailleurs souvent lorsqu'ils étudient $a$ posteriori la première, celle des armes, ou quand ils la vivent dans le moment du présent, que les historiens se trouvent précipités dans la seconde, celle des mots, des idées et des mémoires. Notons qu'à la nature et aux formes que revêt la guerre, s'ajoutent son impulsion et sa dynamique, chacune indissociable de ce qu'elle prend pour cible. Aussi peut-on distinguer la guerre qui vient du dehors, jadis appelée «assaut», «offensive», «incursion», «invasion»..., et à qui le progrès technologique et militaire a fait prendre la forme aussi fulgurante que démultipliée de «frappes». C'est souvent contre la guerre qui vient du dehors que se tourne celle qui part du dedans et qu'on nomme volontiers «résistance» ou «guérilla». Il y a aussi la guerre que le dedans - parfois allié du dehors - se livre à lui-même; on la dit cette fois «soulèvement», «insurrection», «rébellion», «guerre civile». Prise dans ses formes canoniques les plus anciennes comme dans ses formes contemporaines les plus poussées, la guerre trouve sa sanction philosophique et morale, c'est-àdire l'approbation qui la rend exécutoire, dans les qualificatifs aussi divers que sophistiqués dont elle se voit affubler: guerre «juste», guerre «sainte», guerre «punitive», guerre «défensive», guerre «propre», guerre «préventive», etc.

Ces différents aspects et registres de la guerre, qui parfois se chevauchent et s'infléchissent réciproquement, on peut en bonne partie les observer dans ce temps long de l'histoire de Chine qui, à compter du XIX ${ }^{\mathrm{e}}$ siècle jusqu'au milieu du siècle suivant, voit se désagréger le régime impérial et se mettre en place une toute première République, avant que celle-ci ne périclite à son tour. Cette ère de grande instabilité, dont Marie-Claire Bergère souligne qu'elle pourrait tout aussi bien s'appeler le «temps des troubles ${ }^{6}$, se caractérise, on l'aura compris, par un long mouvement de déclin du pouvoir central en Chine, mouvement dont les conflits armés sont à la fois la pierre de touche et le catalyseur. Il suffit de laisser se dérouler le fil des événements pour mesurer l'ampleur de la marche mortifère qui conduit successivement l'empire mandchou et la République de Chine à leur perte.

Les guerres de l'Opium et l'ouverture des ports en Chine par les puissances occidentales à compter des années 1840 , la révolte des Taiping qui secoue le pays de 1851 à 1864 , la défaite cuisante des troupes impériales chinoises face au Japon en 1895, l'échec de la réforme des Cent Jours de 1898, la révolte des Boxers en 1899-1901 sont autant de facteurs et de séquences qui précipitent la chute d'un empire chinois vieux de deux mille ans. L'agonie du régime impérial prend fin avec la révolution de 1911 qui donne naissance à la

6. Voir la préface de l'historienne dans Berger, Bianco et Domes (1989): I-IX. 
République de Chine, proclamée par Sun Yat-sen le 1 janvier 1912. Mais l'idéal émancipateur du nouveau régime se voit aussitôt confisquer par une tentative de restauration monarchique (1912-1916) édictée par le président-général Yuan Shikai. L'épisode, aussi soudain qu'éphémère, se solde à son tour par une désagrégation du pouvoir central au profit de potentats locaux, connus sous le nom de «seigneurs de la guerre» (1916-1927). Il faut attendre l'expédition du Nord (beifa) conduite par Chiang Kai-shek et l'établissement, en 1927, d'un gouvernement nationaliste à Nankin pour que s'amorce - quoiqu'en demi-teinte seulement - une ère de reconquête et d'intégration du pouvoir intérieur. Mais l'unification reste entachée par le conflit entre communistes et nationalistes, auquel s'ajoute, quatre ans plus tard, l'occupation japonaise de la Mandchourie. De 1937 à 1945, l'invasion des troupes nippones dans les régions du Sud et le cortège de violence qui l'accompagne - on pense notamment au massacre de Nankin, perpétré pendant l'hiver 1937-1938 - obligent le gouvernement chinois à se replier dans l'arrière-pays continental, tandis que les provinces du littoral et du Nord tombent sous la férule de pouvoirs collaborateurs ou de guérillas communistes. Et au lendemain de la défaite japonaise de 1945, quand le gouvernement nationaliste cherche à recouvrer sa souveraineté sur le pays, la guerre civile qui l'oppose aux forces communistes tourne à son désavantage. L'unification politique s'accomplit au bénéfice de l'ennemi intérieur, emmené par Mao Zedong, qui fonde le $1^{\text {er }}$ octobre 1949 la République populaire de Chine, obligeant Chiang Kai-shek et ses hommes à se réfugier à Taiwan.

Saisi à l'échelle d'un siècle qui s'étend grosso modo de 1840 à 1949, ce mouvement de l'histoire de Chine se déploie sur fond d'intrusion occidentale bientôt doublée d'une militarisation ravageuse du pays. Mais par-delà les soubresauts et les vicissitudes de l'histoire politique, cette période coïncide aussi avec une ère de grande effervescence sur le plan des idées. À lui tout seul, l'épisode républicain des seigneurs de la guerre frappe par la curieuse similitude qu'il présente en matière de fécondité intellectuelle avec la période des Royaumes Combattants de l'antiquité chinoise tardive (V-III ${ }^{\mathrm{e}}$ siècle av. J.-C.). Non que les idées alors émises ou mobilisées fussent les mêmes, elles se sont entre-temps enrichies de multiples apports - notamment venus d'Occident et du Japon -, mais une fois encore c'est quand le pouvoir central se trouve en pleine déliquescence, et quand rivalisent les ambitions régionales pour l'hégémonie, que fleurissent dans le pays maints courants de pensée, qui bientôt se concurrencent et débattent mutuellement. D'une époque à l'autre, on peut dire de ces courants qu'ils guerroient dans le tumulte des conflits qui les ont vu naître. Les pages qui suivent entendent restituer en partie ces belligérants d'un monde idéel en scrutant la manière dont s'élabore le savoir historien dans la Chine du premier $\mathrm{xx}^{\mathrm{e}}$ siècle. Mais précisons un peu ici ce 
que nous annoncions en ouverture. Cette exploration du travail d'écriture de l'histoire sur fond de guerre consistera principalement à examiner, à partir de l'œuvre de quelques intellectuels et historiens chinois de l'ère républicaine, la façon dont les conflits armés infléchissent la pratique historienne et les possibles mises en récit de l'expérience humaine qui lui sont sous-jacentes. Dans un regard inverse, il s'agira aussi de voir comment, dans le déploiement de la guerre, l'histoire peut, à sa manière, être enrôlée comme instrument de mobilisation sociale, comme récit de positionnement individuel et collectif, ou encore comme outil de légitimation de l'action politique dans le présent.

\section{De la chute de l'empire à une conception nouvelle de l'histoire en Chine: Liang Qichao à l'épreuve de son temps}

Dans son essai «Sur le concept d'histoire», Walter Benjamin avance cette idée selon laquelle l'histoire est toujours écrite par les vainqueurs, suggérant ainsi la prégnance chez celui qu'il nomme l'«historiciste» d'une empathie presque filiale avec les maîtres du moment ${ }^{7}$. En contrepoint de cette idée se profile celle de l'obligation qui est faite aux vaincus, aux opprimés ou aux colonisés de toujours se raccrocher au navire de l'histoire dont seuls leurs subjugueurs tiennent le gouvernail et fixent le cap. Vue sous cet angle, l'histoire se déroberait ainsi toujours aux vaincus en ce qu'elle serait prise tel un butin par les vainqueurs, lesquels, portés par les flots du triomphe, vogueraient vers leurs propres horizons ${ }^{8}$. Le processus de modernisation-occidentalisation de

7. Publié pour la première fois, à titre posthume, en 1942, ce texte est reproduit dans Benjamin (2000): 427-443. Cette version française est due à Walter Benjamin luimême. Notons toutefois ici que c'est son plaidoyer de l'époque pour le matérialisme historique qui vaut au philosophe allemand de pointer du doigt le cortège triomphal d'une histoire toujours écrite par les vainqueurs, histoire que l'historien matérialiste entreprend précisément «de brosser à rebrousse-poil» : «On ne saurait mieux décrire la méthode avec laquelle le matérialisme historique a rompu. C'est la méthode de l'empathie. Elle naît de la paresse du cœur, de l'acedia, qui désespère de saisir la véritable image historique dans son surgissement fugitif. [...] La nature de cette tristesse se dessine plus clairement lorsqu'on se demande à qui précisément l'historiciste s'identifie par empathie. On devra inévitablement répondre: au vainqueur. Or ceux qui règnent à un moment donné sont les héritiers de tous les vainqueurs du passé. L'identification au vainqueur bénéficie donc toujours aux maîtres du moment (432).»

8. Cette image du butin, nous l'empruntons là aussi à Benjamin. Elle revient de façon récurrente dans son essai de 1940 qui dénonçait ainsi déjà à sa manière le «vol» de l'histoire dont nous entretient plus récemment Jack Goody. Voir Goody (2006) (trad. 
l'historiographie qui s'amorce vers la fin du XIX siècle aux quatre coins du monde participe, à bien des égards, de ce phénomène. Si la Chine n'en est point exempte, elle tend cependant à nous dire également autre chose: que le travail d'écriture et de réécriture de l'histoire trouve aussi parfois sa motivation radicale dans les sentiments d'humiliation et les traumatismes qu'engendrent les défaites. Tel est du moins ce qui ressort de l'œuvre et de la réflexion de Liang Qichao (1873-1929), grand lettré de la fin de l'empire et de la jeune République, dont la figure n'est pas sans rappeler celle de nos publicistes européens ${ }^{9}$.

Originaire du district de Xinhui dans le Guangdong, Liang Qichao se lie en tant que disciple au lettré confucéen Kang Youwei (1858-1927) après avoir échoué aux examens impériaux dans la capitale en 1890. Cinq ans plus tard, il compte comme l'un des principaux leaders du mouvement pétitionnaire pour l'annulation du traité de Shimonoseki (gongche shangshu) qui vient de sceller la victoire de l'armée japonaise face à la Chine. Il essuie alors un nouvel échec aux examens impériaux mais décide de rester dans la capitale où il fonde avec son maître en juillet 1895, le Journal des nations (Wanguo gongbao), rebaptisé deux mois plus tard Courrier de Chine et de l'étranger (Zhongwai jiwen). Entre juin et septembre 1898, il prend part à la réforme des Cent Jours pour tenter d'instituer une monarchie constitutionnelle en Chine. Le coup fatal que porte au mouvement la réaction conservatrice à la cour mandchoue l'oblige à prendre le chemin de l'exil pendant quatorze ans, un exil qui le conduira au Japon, où il s'établira principalement jusqu'en 1912, mais aussi en Australie, à Hawaii, aux États-Unis et au Canada.

Le temps des troubles de la période impériale tardive et de l'ère républicaine en Chine traverse tel un fil rouge le parcours, l'œuvre et la réflexion de Liang Qichao, notamment le regard évolutif qu'il porte sur l'histoire. Au lendemain de la défaite mandchoue face aux troupes japonaises, c'est vers les études historiques, en effet, ou plutôt vers leurs modalités d'écriture que se tourne un Liang Qichao désabusé. Frappé de disgrâce et réfugié dans l'archipel nippon après l'échec des réformes dont il s'était fait le fervent promoteur, il publie en novembre 1902 dans les Mélanges sur le renouveau du peuple (Xinmin congbao), tribune d'expression qu'il a lui-même fondée à Yokohama, un essai

française: Le Vol de l'histoire. Comment l'Europe a imposé le récit de son passé au reste du monde, Paris, Gallimard, 2010).

9. Sur la vie et l'œuvre de Liang Qichao, voir les monographies en anglais de Levenson (1959) et de Huang (1972). Parmi une abondante littérature en langue chinoise sur le personnage et sa réflexion, voir l'incontournable biographie que lui consacrent Ding et Zhao (1983); ainsi que Huang (2006). 
au titre programmatique, Renouveler l'historiographie ${ }^{10}$. Le texte s'ouvre ainsi :

De toutes les disciplines du savoir aujourd'hui répandues en Occident, l'histoire est la seule dont la Chine soit pourvue. Et de toutes les disciplines du savoir, l'histoire demeure la plus vaste et la plus importante qui soit. Miroir du pays et du peuple, elle est à la source du sentiment patriotique. Si les nationalismes connaissent un tel essor de nos jours en Europe, si les pays progressent de jour en jour sur le chemin de la civilisation, il faut en rechercher les causes du côté de l'histoire, qui y contribue en bonne part. Aussi bien, la seule chose dont un pays aurait à s'inquiéter serait d'être dépourvu de pareille discipline; on ne voit pas que dans un pays prémuni de celle-ci le peuple ne parvienne pas à s'unir, ni le gouvernement des hommes à se perfectionner. Dès lors, comment expliquer qu'un pays comme le nôtre, qui jouit d'une tradition aussi riche et aussi longue de l'histoire, soit en proie à un tel désastre $^{11}$.

Les raisons du désastre, Liang Qichao estime qu'il faut les rechercher du côté de l'histoire, mais pas n'importe quelle histoire, celle qui s'inscrit dans une tradition immémoriale en Chine, et qui transparaît dans ses formes les plus conventionnelles d'écriture. Ce que Liang Qichao reproche à cette histoirelà, dont il stigmatise les travers dans un premier chapitre intitulé «La vieille histoire en Chine», c'est de n'avoir jamais su cultiver ni instiller dans son pays une sensibilité nationale qui eût permis à celui-ci de tenir tête aux puissances occidentales et au Japon. Liang Qichao dénonce le caractère élitiste de l'historiographie traditionnelle qui, retraçant les seuls faits et gestes de la cour, ne s'adresse qu'au monarque et à la bureaucratie du temps présent. Cette tradition, qui se réduit selon lui à rien de plus que «vingt-quatre généalogies de lignées impériales» (ershisi xing zhi jiapu), «connaît la vie de la cour mais ignore tout de l'État» (zhi you chaoting er bu zhi you guojia). De même, elle

10. Texte reproduit dans Liang (1980a) : 3-40. Cet écrit de Liang Qichao n'a fait l'objet, à ce jour, d'aucune traduction intégrale en langues occidentales. Les travaux en anglais qui le mentionnent ou le citent partiellement traduisent la plupart du temps son titre par «New History » ou «New Historiography ». Nous préférons traduire par «renouveler» le terme xin du titre original, afin de restituer l'acception verbale qu'on lui connaît dans la langue chinoise classique. La rhétorique du renouveau/renouvellement irrigue en effet les écrits de Liang Qichao du début des années 1900. Les Mélanges sur le renouveau du peuple sont eux-mêmes ainsi baptisés par Liang Qichao en référence à l'expression xinmin («renouveler le peuple») qui apparaît en Chine dans l'exégèse confucianiste qui s'élabore au XII siècle autour du livre de la Grande Étude (Daxue).

11. Liang (1980a): 3 . 
«connaît l'action des agents individuels mais ignore le corps social» (zhi you geren er bu zhi you qunti). En ce sens, l'écriture chinoise de l'histoire se refuse à appréhender le peuple comme sujet et comme acteur collectif et conscient. C'est également une complaisance à l'égard des «vestiges du passé» et le refus de penser ou d'observer les «réalités du présent» (zhi you chenji er bu zhi you jinwu) que Liang Qichao dénonce dans le travail des historiographes de l'ère impériale. Cette tendance s'illustre notamment dans l'usage en Chine voulant que ce soit toujours après la chute d'une dynastie qu'on entreprenne d'en relater l'histoire officielle ${ }^{12}$. La tradition historiographique se borne par ailleurs «à la relation des faits et ignore tout effort de conceptualisation » (zhi you shishi er bu zhi you lixiang). Avec l'emphase qui le caractérise, Liang Qichao conclut que sans «une révolution des études historiques» (shijie geming), la Chine ne parviendra jamais à s'extraire de l'ornière politique où elle est embourbée. Aucune voie de salut national, quelle qu'elle soit, ne s'offrira à elle ${ }^{13}$.

Comme le note Nicolas Beaupré, parce qu'elle signale nécessairement l'entrée dans une ère nouvelle et marque ainsi toujours une rupture, la guerre qui éclate tend à être élevée par les hommes, ceux qu'elle implique ou ceux qui la regardent, au rang de mythe fondateur ${ }^{14}$. C'est dans cette perspective, c'està-dire en gardant en ligne de mire ce changement radical intrinsèque à l'entrée dans un monde en guerre, que mérite d'être reconsidéré le réquisitoire de Liang Qichao contre la tradition historiographique en Chine. La réflexion du lettré sur la pratique historienne comme sur le monde d'hier et du présent s'inscrit dans le temps long de l'histoire chinoise, un temps dont on peut dire qu'il est aussi celui des «tentatives de modernité», pour emprunter ici la formulation d'Anne Cheng ${ }^{15}$. Cette quête modernisatrice se trouve précipitée par les conflits successifs dont la Chine sort perdante et ne parvient pas à se relever. Pour les élites lettrées du pays qui portent a posteriori leur regard sur elles, les guerres de l'Opium tiennent en somme du mythe fondateur: elles marquent à leur manière l'entrée dans une modernité chinoise qui introduit une rupture dans l'ordre du discours, des représentations et des croyances. Tant sur le plan intellectuel que sous l'angle culturel ou symbolique, ce moment procède tout

12. Notons que cette pratique perdure jusqu'à la République de Chine, quand, pour tirer un trait sur la dynastie mandchoue antérieure et légitimer son propre régime, le Guomindang met en place en 1914 un Bureau de rédaction d'une histoire officielle des Qing (Qingshi guan). Avant d'être dissous en 1930, le bureau publia en 1928 une Ébauche d'histoire des Qing rédigée dans le plus pur style classique des histoires dynastiques. Sur ce sujet, voir Chen (2006): 173 sqq.

13. Liang (1980a) : 3-9.

14. Voir Beaupré (2006) : 27.

15. Cheng (2003): 179-196, et ici: 181. 
à la fois d'un effondrement des certitudes millénaires, d'un basculement des consciences et d'une crise identitaire profonde. Les désarrois que suscite la tournure prise par les événements en Chine à compter de la seconde moitié du $\mathrm{XIX}^{\mathrm{e}}$ siècle viennent saper les fondements d'un universalisme impérial chinois jusqu'alors si sûr de lui - un universalisme qui voulait que l'ordre du monde et la Civilisation se confondent avec l'empire lui-même. Car, on l'a dit et redit, avec les guerres de l'Opium, ce qui change dans le pays, c'est l'intrusion en force d'un Occident dont la richesse et la puissance des canonnières obligent l'empire à reconnaître désormais «non seulement l'existence mais aussi la supériorité d'un autre que lui et qui n'est pas réductible au statut traditionnel de "barbare des marges" ${ }^{16}$. à l'universalisme impérial se substitue au tournant $\mathrm{du} \mathrm{xx}^{\mathrm{e}}$ siècle en Chine un iconoclasme nationaliste et républicain dans lequel se joue la construction du politique dans sa version moderne - énoncée en termes culturalistes à l'époque.

Le changement qu'induit une telle rupture dans la conscience historique chinoise va de pair avec le développement d'une historiographie nationale rénovée dont Liang Qichao, par son essai de 1902, se fait à la fois l'artisan et le précurseur. En comparaison de ses aînés, tels Gong Zizhen (1792-1841), Wei Yuan (1794-1857), Feng Guifen (1809-1874) ou Sun Jianai (1827-1909), qui vécurent en prise directe la montée des ambitions européennes et les guerres de l'Opium en Chine, Liang Qichao est davantage habité par cette conviction que les causes de la supériorité militaire et économique de l'Occident ne sont pas à rechercher dans un savoir-faire réduit à ses seules dimensions instrumentale et technologique. La force et la richesse des nations occidentales ont selon lui des sources beaucoup plus profondes ancrées dans les évolutions mêmes de la pensée européenne depuis la Renaissance, et en particulier le développement des sciences - auxquelles s'adjoint, plus tard, la naissance des institutions démocratiques. C'est précisément au rang des sciences, notamment celles de la nature, qui ont supposément contribué à la puissace de l'Occident, que Liang Qichao espère parvenir à hisser en Chine les études historiques et la connaissance du fait social, un peu comme ont cru pouvoir le faire avant lui les historiens issus de l'école positiviste allemande, incarnée au premier chef par Ranke (1795-1886). Cette même croyance scientiste guide et informe l'iconoclasme qui s'exprimera bientôt dans le pays à travers le mouvement

16. Ibid.: 182 . 
pour la «Nouvelle Culture » et la séquence symbolique du 4 mai 1919, épisode complexe souvent décrit comme le rêve d'un Aufklärung à la chinoise ${ }^{17}$.

À travers l'appel de Liang Qichao à une révolution des études historiques s'affirme une conception chinoise nouvelle de l'histoire à l'orée du $\mathrm{XX}^{\mathrm{e}}$ siècle. Pour le lettré de la fin de l'empire à la fois rompu à l'étude des Classiques confucéens et initié aux grandes théories du monde contemporain, il est urgent de pouvoir repenser le nouvel ordre mondial qui s'impose à lui et aux siens. Dans son «Autobiographie au seuil de la trentaine», Liang Qichao confesse que ce n'est pas avant l'âge de dix-huit ans, soit en 1890, quand il tomba fortuitement à Shanghai sur une carte du monde établie par le géographe Xu Jiyu (1795-1873), qu'il prit conscience de l'existence sur la planète de cinq continents habités par d'autres pays que le sien, avec leurs populations hétéroclites ${ }^{18}$. C'est ainsi que, douze ans plus tard, il se donne pour tâche de promouvoir une vision de l'histoire et du monde en rupture avec le sinocentrisme de l'historiographie traditionnelle, une vision globale au sein de laquelle la Chine s'insère désormais en tant que nation parmi les autres ${ }^{19}$. L'on mesure bien ici l'ampleur de cet effort de reconceptualisation de l'investigation historique: longtemps considérée en Chine comme le miroir exemplaire de l'expérience humaine, miroir dans lequel se reflétaient les actions des anciens susceptibles d'éclairer la conduite du monarque du temps présent, l'histoire, telle que saisie par Liang Qichao, quitte subitement ses habits de magistra vitae. Elle se définit maintenant comme le lieu d'un récit national, porteur d'une mémoire collective partagée par l'ensemble du peuple qui lui donne corps, et source d'une appartenance commune dans le présent. La conception chinoise traditionnelle de l'histoire, façonnée depuis le XIII siècle par l'influence du confucianisme Song et Ming, tendait trop souvent à cantonner l'observation du passé à sa seule dimension introspective et morale ; elle impliquait un niveau de compréhension de l'expérience humaine sinon anhistorique, du moins frappé du sceau d'une certaine convergence temporelle: ce qui était louable ou condamnable hier l'était tout autant aujourd'hui. La

17. Sur le Mouvement du 4 mai 1919, nous renvoyons ici aux deux ouvrages désormais classiques en la matière de Schwarcz (1986) et Chow (1960). Voir également Goldman et Lee (2002); Lin (1979); ou encore Grieder (1970).

18. Voir Liang (1936d): 16.

19. La monographie que Tang Xiaobing consacre en anglais à Liang Qichao entend précisément sonder l'effort déployé par ce dernier pour repenser l'histoire et le monde, ainsi que les réajustements successifs de ce travail de reconceptualisation. Voir Tang (1996). Sur la méthodologie, la pensée de l'histoire et les travaux historiens dus à Liang Qichao, on trouve également de nombreux éléments dans l'ouvrage de Wang (2001): 42-50; 103-111. 
réflexion historiographique de Liang Qichao est novatrice en ce qu'elle se situe à un double niveau heuristique et patrimonial de compréhension de l'histoire : il s'agit de toucher à la véracité des faits et de parvenir à l'intelligence du passé dont l'héritage et les prolongements sont constitutifs d'une identité nationale au présent ${ }^{20}$.

La définition que donne Liang Qichao des études historiques dans le second chapitre de son essai montre combien sa réflexion est empreinte d'un nationalisme pétri des influences européennes de son temps ${ }^{21}$. Ces apports sont surtout ceux du libéralisme et du darwinisme introduits dès la seconde moitié du XIX ${ }^{\mathrm{e}}$ siècle en Chine, par le biais des traductions de Yan Fu (1853-1921), éminent lettré et ami de Liang Qichao, mais aussi par la médiation des sources secondaires en langue japonaise. Libéralisme dans la mesure où les études historiques prennent chez Liang Qichao la forme non plus de chroniques descriptives focalisées sur l'action des élites dirigeantes et de la bureaucratie impériale, mais d'un récit centré sur le peuple pris dans son ensemble et considéré comme entité une, indivisible et libre de ses choix. Darwinisme dans la mesure où ce récit nouveau de l'expérience nationale s'enracine lui-même dans une conception évolutionniste et linéaire de l'histoire selon laquelle la lutte pour la survie des espèces se voit transposée à l'échelle des nations. Nul besoin de dire ici combien la compétition agonistique que Liang Qichao décèle dans la coexistence des peuples et des nations est exacerbée en Chine par l'arioso des guerres dont l'Occident et le Japon vainqueurs donnent le la. C'est d'ailleurs dans l'expression la plus individuelle de la guerre, la figure du soldat, que Liang Qichao prend la mesure de la formidable avancée qu'a connue sa terre d'accueil japonaise, laquelle souligne en regard l'oligophrénie patente de sa terre ancestrale. Dans ses écrits de 1902, il relate en effet comment, au détour d'une promenade effectuée un beau jour dans le quartier d'Ueno à Tokyo, il eut loisir de croiser sur sa route une parade de soldats japonais tout juste enrôlés dans l'armée. Ces jeunes militaires défilaient dans la rue le regard fier et altier, arborant leurs uniformes neufs, comme autant de bushidō des temps modernes. Leurs banderoles non moins ostensibles où il crut lire en japonais «Prions pour le trépas dans la guerre» l'amenèrent à la conclusion

20. Un article du sinologue Yu Ying-shih montre comment l'invention d'un récit nouveau de l'histoire en Chine enraciné dans le concept de «nation» rompt avec l'historiographie de l'ère impériale, historiographie selon laquelle l'empire chinois embrassait la totalité d'un ordre cosmologique désigné par la notion du «Tout sous le Ciel» (tianxia). Voir Yu (1994): 155-174. Sur cette question, voir également Gray (1961) : 186-212.

21. Voir Liang (1980a): 10-15. 
que l'une des principales faiblesses de son pays natal résidait dans son absence d'esprit guerrier et dans sa peur de l'aventure ${ }^{22}$.

\section{Du progrès triomphant au progrès décadent : la guerre dans le tournant de la pensée historiographique de Liang Qichao}

Pour le Liang Qichao de la fin de l'empire, énoncer le monde au seuil $\mathrm{du} \mathrm{Xx}^{\mathrm{e}}$ siècle, dans tout ce qu'il présentait d'inédit, revenait à formuler celuici en le donnant à lire «saisi par l'histoire, traversé, pétri par elle», si on peut reprendre ici le constat que dresse François Hartog à propos des grands romanciers du XIX ${ }^{\mathrm{e}}$ siècle en Europe ${ }^{23}$. La vision évolutionniste que le lettré chinois cautionnait du cours de l'histoire tranchait avec la conception séculaire en Chine d'un antique passé perçu comme un âge d'or lointain, par essence supérieur au présent. Entraîné par la marche triomphante du progrès, le temps du présent et du lendemain l'emportait désormais sur tout ce qui avait précédé. Synchroniquement appréhendées dans cette puissante et irréversible diachronie du progrès, la civilisation chinoise et la civilisation occidentale se situaient à des stades différents et inégaux de l'histoire qui les privaient de la possibilité d'une modernité partagée. Parce que la première n'avait pas encore atteint le futur olympien de la seconde, elle lui était temporellement et historiquement inférieure.

Quand Liang Qichao fait paraître en 1922 sa Méthodologie de la recherche pour l'histoire de Chine, ouvrage que prolongera un Appendice publié en 1927, c'est, si l'on ose dire, à une toute autre histoire que l'on a affaire à présent ${ }^{24}$. Celle-ci, en effet, n'y est plus perçue comme le lieu d'une convergence universelle et temporelle, sorte d'horizon commun des sociétés inéluctablement poussées vers un avenir substantiellement meilleur. L'histoire est le lieu non plus d'une convergence, mais d'une incommensurable divergence, ou plutôt le lieu de toutes les dissemblances. Elle participe désormais d'un processus culturel global et relatif qui tolère tous les écarts de culture et exclut toute hiérarchie historique. C'est dans la synchronie d'une histoire frappée au coin de la relativité culturelle, prise comme fait «civilisationnel » total pour ainsi dire, qu'il faut maintenant selon Liang Qichao observer la marche diachronique des peuples et des nations. Dans cet ordre des choses, nulle supériorité occidentale, nulle infériorité chinoise : la modernité intervient en des temps et en des lieux

22. Voir Liang (1936a): 37.

23. Hartog (2013): 165 .

24. Voir Liang (1980 b) : 41-177. L'Appendice est reproduit dans Liang (1989). 
différents; elle revêt selon les cultures des formes propres et diverses qui ne répondent à aucun critère qu'un Occident aurait supposément érigé en norme universelle. Dès lors, toute culture ne se différencie que par l'écart qui la sépare de cette autre dont elle se démarque mais qui ne vaut ni plus ni moins qu'elle. La tradition historiographique chinoise vouée aux gémonies par Liang Qichao en 1902 est ainsi remise à l'honneur dans l'ouvrage de 1922, notamment à travers l'éloge de certaines figures d'historiens chinois de l'ère impériale (Liu Zhiji, Sima Guang, Zhao Yi, Zhang Xuecheng...). Dans l'œuvre de ces derniers, Liang Qichao détecte désormais l'existence d'un rationalisme scientifique avant l'heure qui s'illustre notamment à travers une attention constante prêtée à l'étude comparée et à l'analyse critique des sources, ou à la collecte des témoignages sur le terrain. Pourquoi pareil tournant conceptuel dans la pensée historiographique de Liang Qichao? Que s'est-il passé en l'espace de vingt ans?

Que s'est-il passé ? Eh bien, la guerre, là encore. Pas n'importe laquelle, celle que certains «poilus» avaient dès ses premières heures baptisée la «Grande Guerre ${ }^{25}$ et dont Liang Qichao a pu scruter in situ le désastre et les ravages. Tout voyageur aguerri qu'il fût en décembre 1918, notre lettré chinois ne soupçonnait pas le spectacle d'hécatombe et de désolation qui s'offrirait à lui un mois plus tard, lorsqu'il atteindrait les îles britanniques pour arpenter une année durant le continent européen. Les Impressions de voyage en Europe qu'il rédige au fil de ses pérégrinations sur le Vieux Continent, et qu'il publie en $1920^{26}$, montrent que le premier conflit mondial de 1914-1918 - au cours duquel la Chine a fini par rejoindre le camp des Alliés, comme l'appelait de ses vœux Liang Qichao ${ }^{27}$ - n'a pas seulement englouti avec lui les hommes, mais aussi l'adhésion jadis inébranlable à l'idée de progrès. Chez Liang Qichao comme chez ses homologues européens, un tel cataclysme participe de cette crise du régime moderne d'historicité décrite par François Hartog 28 . Le progrès technologique et matériel qu'incarnaient les sociétés industrielles - et leur efficacité à faire la guerre - a en effet conduit à la ruine des nations européennes et à l'effondrement d'une croyance sûre et optimiste en l'Histoire. Le régime moderne d'historicité s'en est ainsi trouvé ébranlé: jusque là prédominé par la temporalité d'un futur pensé comme telos, il donnait à voir un écart croissant entre ces deux métacatégories que l'historien allemand

25. L'historienne Annette Becker retrouve l'expression dans la correspondance de plusieurs poilus dès le mois de novembre 1914. Voir Becker $(1998 ; 2012)$.

26. Voir Liang (1936 b).

27. Voir Liang (1936 c) : 1-76.

28. Hartog (2013): 163-224; 225-285. 
Reinhart Koselleck nomme le champ d'expérience et l'horizon d'attente ${ }^{29}$. L'époque moderne voulait que du futur vînt «la lumière qui éclaire le passé», remarque Hartog. Le temps n'était plus, comme on avait pu l'observer dans l'antiquité, «simple principe de classement, mais acteur, opérateur d'une histoire processus, [...] l'autre nom ou le nom véritable du Progrès ${ }^{30}$. C'est ainsi que, dans l'Europe du XIX ${ }^{\mathrm{e}}$ siècle, on s'était mis à croire en l'Histoire, à croire aussi que les hommes faisaient l'Histoire : «La découverte et la mise en forme d'une histoire processus, régie par le progrès, a correspondu à l'heureux temps, sûr de lui et conquérant, des philosophies de l'histoire, des histoires universelles ou de la civilisation ${ }^{31}$.» Après la Première Guerre mondiale, c'en est fini de cet optimisme et de la croyance en l'histoire-progrès. C'est ce qu'indique la prosopopée bien connue d'un Paul Valéry ${ }^{32}$, ou encore le constat que dressera plus tard l'historien Henri-Irénée Marrou: «[...] au lendemain des grandes tueries de 1914-1918, une illusion s'était dissipée à jamais - la croyance confortable et naïve dans un progrès linéaire et continu qui justifiait la civilisation occidentale comme la dernière étape atteinte par l'évolution de l'humanité ${ }^{33}$.»

Les images d'engloutissement et la vision pessimiste d'une Europe décadente qui informent les Impressions de voyage de Liang Qichao témoignent à leur manière de cette désillusion de l'après-guerre dont le lettré chinois a été le témoin, et à laquelle il souscrit. La description de son voyage à Paris débute par un sinistre tableau de la résidence où il séjourne, laquelle donne sur une cour aux chrysanthèmes fanés, meurtris par un froid automnal qui n'épargne pas non plus les Parisiens «au ventre rationné» et «privés de bois de chauffage ${ }^{34}$. Maintenant que la guerre est consumée, elle laisse place au doute et aux incertitudes. Car elle vient de révéler l'imprédictible nature et l'imprédictible tournure - de l'histoire. C'est du moins ce qui ressort de la prose de Liang Qichao, lorsqu'il écrit:

Quelle étrange créature que cet homme coincé entre ciel et terre! En tout temps, il prend place là où bon lui semble et transforme au gré de sa volonté son propre environnement. Or ne voilà-t-il pas que c'est lui qui, maintenant, se voit gouverner

29. Koselleck (1990) : 307-329.

30. Hartog (2013) : 227-228.

31. Ibid.: 228 .

32. «Nous autres civilisations, nous savons maintenant que nous sommes mortelles...», $c f$. Valéry (1957): 988.

33. Marrou (1968): 15.

34. Liang (1936 b) : 1 . 
par le cadre de vie qu'il a lui-même forgé et façonné. Car ce qu'il produit et transforme, nul ne peut en prédire les conséquences, pas même celui qui s'en faisait l'auteur. Personne parmi nous n'est à même de prévoir la configuration nouvelle qu'engendre l'action productrice et transformatrice de l'homme. Tout le monde est forcé d'en contempler le résultat, la mine déconfite! Alors, sur les fondations de la configuration nouvelle qui s'offre à lui, l'homme doit une fois encore produire et transformer. Toute parcelle d'histoire relève de ce processus dont on ne voit pas où il s'arrête. Qui aurait pu dire, quelques années auparavant, que l'auguste édifice de la Sainte-Alliance, en l'espace d'une dizaine de mois, s'écroulerait comme un seul bloc et se verrait ainsi cloué au sol? Qui aurait pu dire que, dans le même temps, quelque dix monarques trouveraient refuge en Suisse et en Hollande, dans le dénuement le plus total, avec pour seul lot de consolation l'ombre d'eux-mêmes? Qui encore aurait pu dire que l'Union soviétique, celle-là même qu'on surnommait en Orient «le rapace de tous les rapaces », finirait par être amputée de toutes parts et tenue à l'écart de la conférence internationale de la paix à Versailles ? [...] Qui enfin aurait pu dire que l'Angleterre, la France et l'Allemagne, ces trois vieux capitaines de navire, crieraient un jour famine et auraient à vivre dans l'endettement matériel et aux crochets d'autrui, comme cela avait plutôt d'ordinaire été notre cas ${ }^{35}$ ?

Notons que, pour la plupart des penseurs européens contemporains de Liang Qichao, la Première Guerre mondiale se solde en quelque sorte par un arrêt sur image de l'histoire et de la marche du temps, un arrêt qui en fait cruellement apparaître les failles jadis infigurables, ces fameuses «brèches» (gaps) que relèvera plus tard Hannah Arendt ${ }^{36}$. Chez Liang Qichao, en revanche, le moment coïncide avec un «tournant historique de l'humanité»(renlei lishi dezhuanlie). La fin du conflit annonce en effet selon lui, du moins le croit-il à l'époque, l'avènement d'une ère inédite où, face à une Europe mortelle et moribonde, son pays aura une pièce à jouer sur l'échiquier international ${ }^{37}$. Cette vision, ou plutôt cette ré-vision de l'histoire induit une méthodologie nouvelle des études historiques que Liang Qichao formalise dans les six années qui suivent son retour d'Europe, alors qu'il enseigne l'histoire à l'université Qinghua de Pékin. Le professeur concède désormais volontiers que la discipline historique diffère fondamentalement des sciences de la nature. Cette divergence, c'est à l'aune de la catégorie de l'événement que Liang Qichao la mesure. Tandis que les sciences de la nature se fondent sur la récurrence des phénomènes physiques dont elles analysent les lois, les causes et les effets - qu'elles

35. Ibid.: 2-3.

36. Voir Arendt (1972): 11-27.

37. Liang (1936 b) : 3-4. 
peuvent prédire par la suite -, l'histoire, elle, s'enracine dans une concaténation et une accumulation d'événements dont on peut percevoir a posteriori les logiques, mais qu'on ne saurait en aucun cas connaître à l'avance. La nature idiographique et imprévisible de l'événement historique réfute l'idée de toute nomologie applicable au procès de l'histoire ${ }^{38}$.

En 1919, après plusieurs échecs en politique, gagné par le désarroi que suscitait chez lui le spectacle d'une Chine ravagée par les seigneurs de la guerre, la militarisation et la désunion, Liang Qichao mettait les voiles pour l'Europe empli d'une curiosité enthousiaste, tel un passager des mers voguant à la rencontre du futur. Il revient en Chine profondément ébranlé par ce voyage qui lui a fait voir le sombre visage de l'homme occidental. Dès lors, renonçant à toute ambition politique, il se réfugie dans l'étude, et surtout derrière un nationalisme teinté de conservatisme culturel. Non que cette tendance soit nouvelle chez Liang Qichao. L'ensemble de son parcours et de sa réflexion reste marqué par une certaine ambivalence entre l'ouverture aux valeurs occidentales et le repli sur une tradition chinoise jugée moderne en puissance. Dès 1903, la doctrine politique qu'il élaborait dans ses Propos sur le renouvellement du peuple (Xinmin shuo) était pétrie d'une pensée aux accents conservateurs, communautaires et moralisants. Néanmoins, la déception que fait naître chez lui ce voyage dans une Europe ruinée par celui-là même dont il l'avait crue mère, le Progrès, l'invite désormais à rechercher dans les valeurs d'une Chine millénaire la voie d'une alternative culturelle. Quoi de plus beau finalement que le raffinement de la tradition chinoise ? S'il y avait de l'intellectuel moderne dans le lettré de la fin de l'empire, beaucoup du lettré confucéen survivait dans l'intellectuel de la République, pourrait-on dire à son propos pour faire écho au travail d'Yves Chevrier ${ }^{39}$.

38. Voir Liang (1980 b) : 120-136. Comme le remarque Q. Edward Wang, le philosophe allemand Heinrich Rickert, chef de file du néokantisme de l'école de Bade, avec la réflexion duquel Liang Qichao s'est familiarisé dans les années 1920, conforte le lettré chinois dans la nouvelle méthodologie qu'il assigne à la discipline historique. Voir Wang (2001): 109.

39. Voir Chevrier (1984): 81 sqq. L'auteur dit (en parlant de Liang Qichao): «Il y avait un peu de l'intellectuel chez le lettré; beaucoup du lettré survit dans l'intellectuel"nouveau citoyen" (115).» 


\section{Modernisation et révolution : la guerre des armes dans la guerre des récits de Jiang Tingfu et Fan Wenlan}

L'œuvre et la réflexion de Liang Qichao ne sauraient épuiser à elles seules l'élaboration d'un courant moderne des études historiques dans la Chine du premier $\mathrm{xx}^{\mathrm{e}}$ siècle. Toujours est-il que le lettré cantonnais aura posé les jalons d'une historiographie nouvelle dont la génération ultérieure des intellectuels et historiens du Mouvement du 4 mai 1919 achèvera de définir les pourtours. Ce parachèvement correspond à un moment qu'on peut qualifier de «méthodique» de l'historiographie chinoise contemporaine: les méthodes héritées des sciences occidentales, la collecte des documents de première main, la critique textuelle et l'observation empirique se voient désormais ériger en piliers du travail de recherche historique en Chine. Tels sont du moins les grands préceptes que condense le credo scientifique de l'intellectuel moderniste $\mathrm{Hu}$ Shi (1891-1962): «De l'audace dans l'hypothèse, de la minutie dans la preuve (dadan jiashe, xiaoxin qiuzheng).» Ainsi, dans une collection d'essais en huit volumes éditée entre 1926 et 1941 par l'historien Gu Jiegang (1893-1980) sous le titre de Discussions critiques sur l'histoire ancienne (Gushi bian), l'application de la critique historique occidentale au corpus traditionnel de la littérature pré-impériale est radicalisée à l'extrême. On y remet en cause l'authenticité de la plupart des textes anciens, de même qu'on y révise à la baisse les datations communément admises ${ }^{40}$. Mêlant positivisme et érudition pure, l'école dite de la «documentation historique», ou école du shiliao (shiliao xиepai), qui voit le jour en 1928 à l'instigation de l'historien Fu Sinian (1896-1950), s'inscrit elle aussi dans cette mouvance critique des études historiques. Fu Sinian en énonce les préceptes au moment où il fonde en 1928 ce qui deviendra une institution phare de la recherche en histoire dans la Chine républicaine - et à Taiwan après 1949 -, l'Institut d'histoire et de philologie, rattaché deux mois après sa création à l'Academia Sinica ${ }^{41}$. En récusant toute forme d'interprétation spéculative et morale du cours de l'histoire, le courant dont se réclame Fu Sinian et ceux qui lui sont proches prend le contre-pied de l'historiographie traditionnelle d'inspiration néoconfucéenne. Il rejette

40. Sur Gu Jiegang et ce mouvement de remise en cause radicale de l'historiographie traditionnelle de la Chine ancienne, voir Schneider (1971). Voir aussi la traduction annotée par le missionnaire américain ArthurW. Hummel de la préface autobiographique que Gu Jiegang apporte au premier volume du Gushi bian: Hummel (1931). Voir enfin Wang (1987).

41. Sur la vie et l'œuvre de Fu Sinian, voir la monographie de Wang (2000). Voir aussi la section qui lui est consacrée dans Morier-Genoud (2014): 249-261. 
aussi la tendance marxiste alors naissante en Chine, tendance que le sinologue Yu Ying-shih range, par opposition à l'école du shiliao, sous la bannière de l' «école de la conception historique» (shiguan xuepai) ${ }^{42}$. Introduite en 19181920 par l'activiste communiste Li Dazhao (1888-1927), mais également par deux théoriciens droitiers du Guomindang, Hu Hanmin (1870-1936) et Dai Jitao (1891-1949), la théorie marxiste de l'histoire emporte bientôt l'adhésion de certains intellectuels révolutionnaires qui tentent de la mettre en pratique, tels Fan Wenlan (1893-1969), Qu Qiubai (1899-1935), Li Da (1890-1966) ou Cai Hesen (1895-1931), pour ne citer qu'eux ${ }^{43}$. Ces derniers trouvent en elle une puissante sociologie du changement qui, en même temps qu'elle place la société au point de départ de l'investigation historique, cherche dans les processus sociaux les forces supposées façonner l'histoire. Ce regard nouveau sur la société, le monde et l'histoire introduit une rupture épistémologique avec le modèle traditionnel des histoires officielles (zhengshi) et des chroniques locales (fangzhi). Les premières comme les secondes tendaient en effet à focaliser l'attention sur l'action des hommes jugés influents dans l'histoire. Désormais, les agents individuels se voient dissous dans des entités impersonnelles qui dépassent la seule sphère de l'individu et dont la prise en compte rend caduque la tendance même de l'historiographie traditionnelle à l'hagiographie. Cette dissolution procède du déplacement de l'objet de l'histoire: les personnages naguère tenus pour héros de l'action historique se trouvent subsumés sous l'action de nouvelles forces - sociales, économiques, culturelles - dont il s'agit à présent de saisir les logiques, les dynamiques et les structures.

Avec l'œuvre et la réflexion des historiens Jiang Tingfu (1895-1965) et Fan Wenlan (1893-1969) s'illustrent deux grands récits paradigmatiques de l'histoire chinoise moderne que le spécialiste Li Huaiyin analyse avec force acuité: celui de la «modernisation» et celui de la «révolution ${ }^{44}$. Dans la Chine républicaine des années 1930-1940, ces deux récits s'inscrivent en rupture en même temps qu'ils s'amplifient mutuellement. L'un et l'autre sont à

42. Par «conception historique» (shiguan), Yu Ying-shih fait ici allusion au déterminisme et à l'apriorisme qui sous-tendent la théorie marxiste de l'histoire. Pour le sinologue, le courant marxiste cautionne en effet une conception et un modèle explicatif préétablis de l'histoire dans la mesure où il postule, en amont de toute observation empirique des faits, la validité du concept de matérialisme historique. Voir Yu (1982) : 7-26.

43. Sur l'introduction de la théorie marxiste de l'histoire en Chine, voir Dirlik (1978); et Brook (1990) : 110-157. Pour ce qui est de la contribution à l'histoire des intellectuels révolutionnaires dont nous énumérons les noms ici, nous renvoyons à Wu (2002) : 21143.

44. Voir Li (2013): 33-73; 74-109. 
rattacher respectivement à une mouvance d'inspiration libérale et à un courant maoïste de l'historiographie chinoise contemporaine. La question des conflits armés leur est centrale. D'abord parce que les guerres du présent, notamment la confrontation sino-japonaise, invitent leurs auteurs respectifs à réévaluer les guerres du passé en Chine, dont ils cautionnent des lectures antagonistes. Ensuite parce que les conflits du moment ne sont pas sans conséquence sur l'attitude de ces derniers vis-à-vis du pouvoir nationaliste chinois, ni non plus sur le positionnement qu'ils accréditent ou condamnent des acteurs politiques face à l'ennemi d'alors.

Pour ce qui est du récit de la modernisation, il ne saurait se réduire au seul travail de l'historien Jiang Tingfu. À compter du dernier tiers du XIX $X^{e}$ siècle, la modernisation s'est imposée comme un thème lancinant chez les élites lettrées en Chine. Celles-ci l'ont tantôt assimilée à une occidentalisation - plus ou moins partielle - de la pensée et des pratiques dans leur pays, tantôt lui ont cherché une voie de mise en œuvre proprement chinoise. Dans les années 1930 et 1940 , nombre de collègues, amis, disciples et autres penseurs contemporains de Jiang Tingfu souscrivent à cette idée phare de la modernisation, quelles qu'en soient les variantes ${ }^{45}$. Néanmoins, c'est à Jiang Tingfu que l'on doit, à la suite de l'historien Chen Gonglu (1900-1966), une Histoire de la Chine moderne, parue pour la première fois en 1939, qui marquera en profondeur les esprits et les travaux historiens sous la République, et ce même bien après que celle-ci aura trouvé refuge à Taiwan ${ }^{46}$.

Né en 1895 dans un milieu rural relativement modeste du Hunan, Jiang Tingfu se forme aux États-Unis entre 1911 et 1923, année où il obtient un doctorat en histoire à l'université Columbia. De retour en Chine, il enseigne d'abord à l'université Nankai de Tianjin, avant d'être nommé professeur à la faculté d'histoire de l'université Qinghua à Pékin. À la fin des années 1920, Jiang Tingfu prend part au grand projet de collecte et de classification des archives impériales mandchoues que l'historien Fu Sinian supervise à l'Institut d'histoire et de philologie de l'Academia Sinica. Il circonscrit alors son travail à l'étude des documents diplomatiques de la cour des Qing, ce qui lui vaut de

45. À cet égard, l'historien Luo Rongqu affirme - non sans une pointe de chauvinisme que le concept même de modernisation est apparu en Chine environ vingt ans plus tôt qu'en Occident, avant même qu'il ne soit mobilisé par les sciences sociales américaines des années 1950. Voir Luo (1993): 365.

46. Les Commercial Press de Shanghai sont à l'origine de cette première édition, laquelle est intégralement reproduite dans le «Minguo congshu, II» (Grande Collection des œuvres de la période républicaine en Chine, seconde édition). Voir Jiang (1989b). C'est la présente édition que nous citons ci-après. Sur la réception et l'influence de l'ouvrage, y compris à Taiwan après 1949, voir Liu (1983): 87 sqq. 
publier en 1931 deux volumes d'une Synthèse des matériaux sur l'histoire de la politique étrangère de la Chine moderne ${ }^{47}$. L'année suivante, il fonde avec Hu Shi et Ding Wenjiang la revue Duli pinglun (La Critique indépendante). Jusqu'en 1937, cette tribune d'expression fera entendre la voix des intellectuels chinois du 4 mai qui s'opposent au marxisme et affichent leur attachement à l'idéal des démocraties libérales ${ }^{48}$.

Dans la perspective de Jiang Tingfu, bien que l'avancée des sciences et leur application constituent la pierre angulaire du processus de modernisation, elles ne lui sont en rien exclusives. Le phénomène renvoie à des pans multiples de l'expérience humaine, notamment parce que le recours aux sciences modernes dans le secteur de la production transforme toutes les strates de la société et l'ensemble des rapports humains qui la fondent. Tenant pour acquis les bienfaits de la société industrielle et l'accessibilité des nations à une modernité universelle, le récit de la modernisation relève chez Jiang Tingfu d'un processus évolutif et modernisateur qui, depuis la fin du XIX ${ }^{\mathrm{e}}$ siècle, se caractérise en Chine par des tentatives réitérées d'adoption des idées, des techniques et des institutions occidentales. Mais pour multiples qu'elles aient été, et si grande que fût l'ardeur à la tâche de leurs promoteurs, ces entreprises de modernisation durent toujours avorter. L'histoire de la Chine moderne, telle que la voit Jiang Tingfu, se déploie comme une série d'échecs et de frustrations imputables aux barrières culturelles et politiques inhérentes à la tradition et à la société chinoises. Selon Jiang Tingfu, les réformateurs de la fin de l'empire n'ont pas su surmonter ces obstacles dans lesquels convergeait une raison d'être séculaire et proprement chinoise du monde.

Contrairement au récit de la révolution d'un Fan Wenlan qui attribue le retard technologique et économique de la Chine à des facteurs externes - notamment à l'intrusion de l'impérialisme occidental et au système d'exploitation capitaliste qui en découle -, Jiang Tingfu soutient que c'est en raison de facteurs internes que le pays n'a pas su prendre le train de la modernisation. Le Mouvement d' «autorenforcement» (ziqiang) promu par les bureaucrates réformateurs de l'empire dans les années 1860-1890 s'est soldé par un échec retentissant en raison de la compréhension limitée que ces derniers avaient de la civilisation occidentale. En outre, les institutions politiques préexistantes en Chine et les milieux sociaux traditionnels faisaient entrave à tout effort de modernisation. La victoire japonaise de 1895 a révélé combien ce mouvement limité aux

47. Là aussi, la première édition de ce travail, due aux Commercial Press de Shanghai, est reproduite dans le «Minguo congshu, II». Voir Jiang (1989a).

48. Sur le parcours de Jiang Tingfu, voir les mémoires qu'il rédige à la fin des années 1940 : Jiang (2003). Voir aussi Li (2013) : 41-73. 
seuls domaines de la défense et de l'industrie était voué au fiasco ${ }^{49}$. Quant à la réforme des Cent Jours de Kang Youwei et de Liang Qichao, qui visait à l'établissement d'une monarchie constitutionnelle en terre chinoise, elle s'est heurtée au conservatisme particulièrement vif et tenace de la cour et de la bureaucratie traditionnelle ${ }^{50}$. Nonobstant les échecs répétés du passé, Jiang Tingfu reste convaincu que c'est à une élite éclairée qu'il incombe de mener à bonne fin, par la seule voie de la réforme, la modernisation du pays. Celle-ci requiert l'existence d'un État fort, centralisé et unifié, que seul le Guomindang de Chiang Kai-shek lui semble à même de mettre en place. Ceux qui se sont jadis frottés à une telle entreprise réformatrice ont malheureusement dû composer avec une élite dirigeante qui croyait fermement en l'existence d'une prétendue supériorité culturelle chinoise. Refusant de traiter les puissances occidentales sur un pied d'égalité avec la Chine, la cour impériale cautionnait encore la logique d'un système tributaire selon lequel une Chine civilisée et suzeraine nouait contact avec des États vassaux et barbares supposés se mettre à l'école d'un centre civilisateur incarné en la personne de l'empereur. Cette conception du politique se situait aux antipodes des codes et de l'étiquette de la diplomatie moderne. Jiang Tingfu blâme ainsi le refus obstiné du pouvoir impérial d'apprendre de ces «barbares», qu'il fallait tantôt «éliminer» (jiao $y i)$ tantôt «pacifier» $(f u y i)^{51}$.

L'approche élitiste du processus modernisateur à laquelle souscrit Jiang Tingfu diverge fondamentalement du récit de la révolution de Fan Wenlan, récit selon lequel, trouvant sa force motrice dans les antagonismes de classes, le mouvement de progrès et d'émancipation nationale en Chine part du bas de la pyramide sociale. Né en 1893 dans une famille de lettrés du Zhejiang affiliés à l'école du Zhedong, Fan Wenlan est profondément marqué dans son enfance par les humiliations que les dignitaires et les militaires mandchous font subir aux siens et aux couches les plus pauvres de la population han. Dans un texte de 1956, il raconte comment, enfant, il assista en public à l'arrestation et à l'exécution sommaire, par l'armée impériale, de Qiu Jin (1875-1907), célèbre figure féminine de l'activisme révolutionnaire antimandchou et fondatrice d'une école de district où son frère venait parfaire au quotidien sa connaissance des Classiques ${ }^{52}$.

Formé aux «études nationales » (guoxue) à l'université de Pékin par le lettré Huang Kan (1886-1935), Fan Wenlan s'illustre à l'analyse philologique dans

49. Voir Jiang (1989 b) : 62-69.

50. Ibid.: 98-102.

51. Ibid.: 6-7.

52. Voir Fan (1956): 20-22. 
un commentaire du célèbre Wenxin diaolong (L'Esprit des lettres et la gravure des dragons) qu'il publie en 1925, après avoir enseigné pendant trois ans à l'université Nankai de Tianjin. Tandis qu'il poursuit sa carrière d'enseignant à Pékin, il se rapproche du Parti communiste, notamment de l'activiste révolutionnaire Li Dazhao. Il prend part aux grands mouvements étudiants contre l'impérialisme japonais, ce qui lui vaut d'être arrêté à plusieurs reprises par le Guomindang entre 1927 et 1934. Après l'incident du pont Marco Polo en juillet 1937 et le début de la guerre sino-japonaise, Fan Wenlan prône plus que jamais la lutte armée contre le Japon. Il s'exile aux côtés de ses camarades de l'université du Henan dans la région montagneuse de Jigongshan, avant de rejoindre les rangs de la Nouvelle Quatrième Armée communiste. En septembre 1938, il entre au PCC et se réfugie à Yan'an où il se lie d'amitié avec Mao Zedong, qui bientôt le promeut historien officiel du parti. En nommant Fan Wenlan à la tête de la Division des études historiques de l'Institut de Yan'an du marxisme et du léninisme, Mao Zedong n'attend pas seulement du philologue révolutionnaire qu'il donne une assise scientifique et historique au combat idéologique contre l'ennemi nationaliste chinois. Il cherche aussi à contrebalancer l'influence de deux historiens du parti qui souscrivent, sans y déroger, à la conception stalinienne de l'histoire, Zhang Wentian (1900-1976) et Li Dingsheng (1907-1966). L'un et l'autre supervisent alors le Bureau de la propagande du PCC et ne partagent pas la même ligne politique que lui, ni non plus la même vision du mouvement révolutionnaire. Zhang Wentian et Li Dingsheng se posent en tenants d'une orthodoxie marxiste qui voit dans la révolution agraire (tudi geming) la clé de voûte de l'émancipation d'une société chinoise jugée «semi-féodale» (ban fengjian de) et «semi-coloniale» (ban zhimin de). Cette appréciation contrarie l'ambition maoïste d'alors de prendre appui sur l'ensemble des forces sociales, y compris la bourgeoisie nationale et la classe des propriétaires fonciers, pour conduire en Chine, sous la tutelle du prolétariat, une «nouvelle révolution démocratique [et totale]» (xin minzhuzhuyi geming) ${ }^{53}$.

53. À l'époque, Zhang Wentian et Li Dingsheng ne s'opposent pas seulement à la ligne maoïste au sein du parti, mais aussi au courant trotskyste. Les appréciations divergentes que les uns et les autres portent sur la nature de la société chinoise les opposent sur la question de l'orientation à donner au mouvement révolutionnaire. La tendance trotskyste soutient ainsi pour sa part que, la Chine étant devenue une société capitaliste, l'heure a sonné d'une révolution socialiste visant à éradiquer le capitalisme qui la gangrène. Sur ces questions, voir Weigelin-Schwierdrzik (2007) : 3-6. Voir aussi Dirlik (2001): 57-94; et Li (2013) : 95-104. 
Mao Zedong enjoint ainsi Fan Wenlan de s'atteler à la rédaction d'un manuel d'histoire de Chine à l'adresse des membres du parti et des générations futures. Entre le mois d'août 1940 et la fin de l'année 1941, Fan Wenlan travaille à la mise en forme d'un Précis d'histoire générale de Chine (Zhongguo tongshi jianbian) en deux volumes ${ }^{54}$. En mai 1943, il entreprend de rédiger un troisième volet couvrant la période moderne depuis les guerres de l'Opium jusqu'au soulèvement des Boxers. Achevé en 1945, ce dernier tome paraît l'année suivante sous le titre d'Histoire de la Chine moderne ${ }^{55}$. Outre l'impératif de donner au mouvement révolutionnaire une assise populaire, la haine que Fan Wenlan voue aux Mandchous depuis sa plus tendre enfance explique l'enthousiasme qui transparaît dans cet ouvrage lorsqu'il y relate les mouvements antimandchous du peuple chinois, tels la révolte des Taiping et l'épisode des Boxers. L'histoire de la Chine moderne est vue par Fan Wenlan comme le lieu d'un combat séculaire entre des forces révolutionnaires et des forces réactionnaires qui trouvent leur incarnation respective dans le présent, à travers l'action du Parti communiste et celle du Guomindang. La glorification, dans le récit de Fan Wenlan, des rébellions populaires et de leurs leaders, loués comme autant de héros moralement irréprochables, tranche avec la vision historique de la modernisation d'un Jiang Tingfu qui voit dans les couches les plus basses de la société chinoise une populace ignorante et illettrée, rétive au progrès, entravée par ses liens de loyauté envers la famille et les communautés locales. Là où, chez Fan Wenlan, les soulèvements populaires s'inscrivent dans une trajectoire révolutionnaire porteuse de progrès et d'émancipation sociale, ils correspondent, chez Jiang Tingfu, à des périodes régressives de troubles et de chaos faisant obstacle à l'action des élites réformatrices en Chine, et exacerbant l'immixtion des puissances occidentales dans les affaires intérieures du pays.

Jusqu'en 1936, c'est surtout dans l'optique de légitimer la politique de conciliation et d'accommodement du gouvernement nationaliste chinois envers l'agresseur japonais que Jiang Tingfu cherche à promouvoir son récit de la modernisation en Chine. C'est là qu'intervient la question de la guerre dans le travail d'interprétation de l'historien. Car à l'inverse d'un intellectuel et activiste révolutionnaire comme Fan Wenlan, Jiang Tingfu estime que la Chine

54. Ces deux volumes furent édités pour la première fois par l'Institut de Yan'an du marxisme et du léninisme. Le premier parut en septembre 1941 et le second l'année suivante. Ils firent l'objet d'une seconde édition dès 1947 à Shanghai (éd. Xinzhi shuju) avant d'être régulièrement réédités sous la République populaire de Chine. On peut retenir ici l'édition révisée et augmentée par l'auteur: Fan (1989).

55. Voir Fan (1992). 
n'est pas en mesure de résister au Japon après que celui-ci s'est emparé du contrôle de la Mandchourie en 1931. Selon l'historien, le retard technologique et militaire de la Chine condamne le pays, sur la scène interétatique, à la concession et au renoncement, excluant de fait chez lui l'option de la lutte armée contre l'occupant japonais. L'heure n'est pas à la résistance par les armes, mais à la poursuite des réformes et des efforts de modernisation sous la tutelle du Guomindang. Cette tâche doit passer par une centralisation accrue de l'État et par la mise au pas des provinces, c'est-à-dire par la suppression de toute velléité chinoise d'hégémonie locale ou régionale. Jiang Tingfu fustige l'attitude des communistes et activistes du mouvement révolutionnaire des années 1930 qui cherchent à galvaniser les masses laborieuses dans leurs appels à la résistance armée contre les forces japonaises. Dans un texte paru en avril 1933, «Le territoire non occupé est notre échappatoire», l'historien soutient que «la lutte contre les bandits intérieurs» (comprendre le Parti communiste) vient «avant celle contre le Japon». «Aujourd'hui encore, poursuit Jiang Tingfu, certains d'entre nous croient pouvoir s'appuyer sur des armées faites d'épées et de sabres pour se mesurer à des contingents de canons et d'avions. Ne faut-il pas voir ici une croyance aveugle, vaine et désuète? [...] Pour sauvegarder les apparences et ne pas perdre la face, d'aucuns vont même jusqu'à se conforter dans cette idée que des agneaux peuvent combattre des chiens et des fauves ${ }^{56}$.»

C'est dans l'histoire de la Chine moderne que Jiang Tingfu puise les arguments de son engagement politique et trouve la justification de ses prises de position dans le présent. L'historien condamne l'attitude de la frange la plus conservatrice de la bureaucratie de la fin de l'empire qui croyait pouvoir exciter la volonté populaire et s'en remettre au soutien des masses pour supprimer les «barbares », sans tenir compte de l'état d'arriération militaire du pays. La figure de Lin Zexu (1785-1850), célèbre fonctionnaire chargé par le cour mandchoue de mettre un terme au commerce de l'opium imposé par les Anglais, n'échappe pas à cette tendance. Lin Zexu avait bien conscience, estime Jiang Tingfu, de l'incapacité des troupes impériales à tenir tête aux canonnières britanniques. Il savait que le temps était venu pour les siens d'apprendre des Occidentaux. Néanmoins, pour préserver sa réputation de patriote et éviter toute réaction conservatrice et xénophobe à la cour, il affectait, au mépris de ses convictions les plus intimes, une volonté dure comme fer de combattre les Anglais, une volonté qui bénéficiait soi-disant de l'assentiment du peuple chinois. Jiang Tingfu raille l'attitude de Lin Zexu qui, selon lui, n'est pas sans rappeler celle des anciens fonctionnaires impériaux gonflés de vanité, pleins de vantardise ou de projets chimériques, toujours prompts à

56. Jiang (1971 b): 288 . 
proférer de belles paroles (changgaodiao) devant des masses ignorantes qu'ils tenaient acquises à leur cause ${ }^{57}$. Pour Jiang Tingfu, en incitant la population à prendre les armes contre le Japon, les activistes communistes des années 1930 ne font que perpétuer naïvement cette antique fumée de gloriole. À l'inverse, le personnage de Borjigit (1790-1854), Qishan de son nom chinois, qui succéda à Lin Zexu en tant que gouverneur général du Guangdong et du Guangxi, est porté aux nues par Jiang Tingfu, qui l'élève en modèle de réalisme et de lucidité ${ }^{58}$. À l'issue de la première guerre de l'Opium, Qishan fut désavoué et condamné à mort par l'empereur (une peine finalement commuée en exil quelques mois plus tard) pour avoir retiré les troupes impériales des forteresses côtières du Guangdong, et pour avoir signé avec les Anglais, le 20 janvier 1841, sans attendre l'approbation officielle de la cour, la convention de Chuanbi laquelle prévoyait notamment la cession à perpétuité de l'île de Hongkong aux Britanniques. Pour Jiang Tingfu, Qishan n'est aucunement responsable de la défaite chinoise face aux Anglais. C'est l'incapacité technologique et militaire de l'empire à combattre ces derniers qui explique l'inévitable reddition de la cour à l'époque. L'attitude conciliante de Qishan à l'égard des Britanniques et ses efforts déployés en diplomatie étaient justement cela même qui marquait sa prééminence sur la plupart de ses homologues mandchous ${ }^{59}$.

Le contraste est saisissant entre l'appréciation qu'émet Jiang Tingfu à l'endroit de Lin Zexu et Qishan et le portrait que dresse Fan Wenlan des deux protagonistes dans son Histoire de la Chine moderne. À l'opposé de la figure d'un aristocrate comme Muzhang'a (1782-1856), décrié par Fan Wenlan pour avoir collaboré avec les Anglais et s'être enrichi dans le commerce immoral de l'opium, Lin Zexu apparaît dans le récit de l'historien communiste comme un «héros national» (guojia yingxiong) plein de droiture et de vaillance, ayant refusé toute compromission avec l'ennemi britannique lors même qu'un Qishan, «capitulard et traître à son pays» (fangshou maiguo), allait, lui, lâchement s'incliner ${ }^{60}$. «Cent ans après les guerres de l'Opium, écrit Fan Wenlan, une clique réactionnaire [comprendre Chiang Kai-shek et ses hommes] épouse encore dans notre pays la ligne collaborationniste d'un Muzhang'a, confondant de façon éhontée le vrai et le faux, adulant ainsi des collaborateurs qu'elle érige en patriotes lucides, soi-disant conscients de leur propres limites et du poids de l'ennemi d'en face ${ }^{61}$.» Dès les premières pages de son Histoire

57. Voir Jiang (1989b): 29; et Jiang (1971b): 82.

58. Voir Jiang (1971a): 37-61.

59. Ibid.: 51-57.

60. Voir Fan (1992): A15-A40.

61. Ibid.: A32. 
de la Chine moderne, Fan Wenlan rattache l'action de Chiang Kai-shek et du Guomindang à la ligne collaborationniste des réformateurs de la fin de l'empire. D'une époque à l'autre, tous se sont d'après lui employés à jeter la Chine en pâture aux puissances coloniales étrangères. «L'histoire chinoise du siècle dernier, ajoute plus loin Fan Wenlan, n'est rien d'autre que celle d'un peuple en lutte contre ses propres capitulards et ses maîtres impérialistes ${ }^{62}$.»

Replacés dans le contexte politique et historique où ils sont proférés, les propos de Fan Wenlan n'ont pas d'autre visée que de servir la propagande communiste. Ils méritent cependant d'être nuancés. La passivité face à l'ennemi japonais n'a pas été, comme le laisse entendre l'historien révolutionnaire, le seul fait du gouvernement nationaliste chinois de Chiang Kai-shek. Entre 1931 et 1935, remarque Alain Roux, les communistes avaient suivi une politique rigoureusement symétrique à celle du Guomindang, estimant qu'il fallait d'abord renverser Chiang Kai-shek avant de prendre les armes contre le Japon. C'est seulement à compter du mois d'août 1935, sous l'influence du $\mathrm{VII}^{\mathrm{e}}$ congrès de l'Internationale communiste, que la lutte antifasciste, épaulée par les bourgeoisies nationales, était passée à l'ordre du jour et que le PCC avait réajusté sa propagande pour promouvoir ouvertement la résistance antijaponaise $^{63}$. À partir de 1935, l'historien Jiang Tingfu change lui aussi son fusil d'épaule. Conscient de l'indignation que suscite dans l'opinion chinoise l'attentisme du Guomindang, et face à une guerre jugée à présent imminente contre le Japon, Jiang Tingfu s'engage de plain-pied sur le front de la vie politique. Alors même qu'il semblait être au faîte de sa carrière académique, il renonce à ses fonctions universitaires pour occuper différentes charges officielles au sein du gouvernement de Chiang Kai-shek. Après 1937, la lutte armée contre le Japon et le Second Front uni avec les communistes figurent sur son agenda politique. Le libéralisme démocratique des premières heures qu'il prônait avec ses camarades de la Critique indépendante n'est plus l'antidote aux maux du pays. Le salut de la nation passe désormais par la suppression de l'ennemi japonais, l'unification territoriale et une centralisation du pouvoir aux accents étatistes et dirigistes.

On voit ici combien le conflit sino-japonais et la rivalité entre communistes et nationalistes sont au cœur des instrumentalisations de l'histoire chinoise par Jiang Tingfu et Fan Wenlan. La guerre narrative qui fait rage entre les deux historiens trouvera de fait sa résolution dans la fondation de la République populaire de Chine en 1949. Seul le récit de la révolution de Fan Wenlan survivra à la mise en place du régime maoïste sur le continent, tandis que

62. Ibid.: A65.

63. Voir Roux (2004): 68. 
celui de la modernisation d'un Jiang Tingfu s'exportera à Taiwan. Notons que la sinologie occidentale souscrira elle-même pendant longtemps, du moins jusqu'à la fin des années 1980, tantôt à l'un tantôt à l'autre de ces grands récits paradigmatiques et antagonistes de l'histoire de la Chine moderne dont Jiang Tingfu et Fan Wenlan auront été deux éminents artisans sous la République de Chine ${ }^{64}$.

\section{De l'érudition désintéressée à l'étude engagée : Fu Sinian face à la guerre}

La guerre sino-japonaise n'est pas non plus sans conséquence sur le travail de l'historien Fu Sinian. Elle modifie chez lui son rapport à l'étude et au pouvoir, ou plutôt elle annule la distance qu'il entendait mettre entre le scientifique et le politique. Pour le père fondateur de l'Institut d'histoire et de philologie de l'Academia Sinica, le travail historien s'était toujours défini comme la quête d'une réalité intacte et objective. Ceux qui se frottaient à cette entreprise se devaient de cultiver le goût de l'étude pour l'étude. En 1928, Fu Sinian mettait ainsi en garde ses collègues et disciples contre toute compromission des études historiques avec la vie politique et les idéologies partisanes. Cette posture visait surtout à faire pièce au courant des historiens marxistes pour qui la discipline historique devait épouser l'agenda politique du mouvement révolutionnaire ${ }^{65}$. À la suite de l'historien Gu Jiegang et de son maître $\mathrm{Hu}$ Shi, Fu Sinian estimait pouvoir concilier le positivisme de l'historiographie allemande d'un Leopold von Ranke avec la forme d'un rationalisme proprement chinois s'illustrant à partir du XVIII ${ }^{\mathrm{e}}$ siècle, à travers la tradition philologique dite «des vérifications et des preuves», ou kaozheng ${ }^{66}$. Parce que l'histoire

64. On constate en effet aujourd'hui une tendance dans la sinologie américaine des années 1960 et 1970 à épouser le récit de la modernisation chinoise, laquelle y était essentiellement perçue comme une réponse nationale (et nationaliste) à l'agression occidentale en Chine. C'est ce qui ressort, par exemple, de la monographie de Levenson (1959), de même que de l'ouvrage de Black (1966), ou encore du volume édité par Rozman (1981). Du côté français, l'étude que l'on doit au sinologue Jean Chesneaux (1976) peut quant à elle être rattachée au récit de la révolution d'un Fan Wenlan, notamment par l'attention constante qu'elle prête aux révoltes populaires en Chine, décrites comme le moteur du mouvement révolutionnaire chinois.

65. Voir Wang (2000) : 79-81.

66. Au risque d'un certain schématisme, disons que le courant du kaozheng voit le jour en Chine en réaction à une pensée morale et spéculative héritée des Song (960-1279) et des Ming (1368-1644). Il marque un tournant philologique dans l'histoire intellectuelle 
s'enracinait dans le travail d'écriture et dans la narration, elle n'échappait pas selon $\mathrm{Fu}$ Sinian à une mise en intrigue d'elle-même qui la détournait de la réalité historique. Aussi était-ce par la collecte des matériaux de première main et par la critique textuelle - assortie d'une recherche archéologique de longue haleine - que l'historien pouvait supposément toucher à la véracité des faits. Cette délimitation des études historiques, qui refusait de concevoir l'histoire comme le lieu de l'avènement d'une vérité philosophique et morale, récusait l'attitude des historiens marxistes cherchant à retirer de la narration historique un profit politique.

Dès le début de l'occupation japonaise de la Mandchourie, l'esprit d'indépendance et d'impartialité avec lequel Fu Sinian entendait conduire la rechercheenhistoire dans son pays s'en trouvealtéré.L'académisme etl'érudition désintéressée qu'il prônait dans les années 1920 s'effacent maintenant devant la nécessité d'une étude engagée. Le besoin d'œuvrer pour le renforcement et la cohésion d'un sentiment culturel et d'un sentiment patriotique chinois, condition indispensable au salut national, s'impose à $\mathrm{Fu}$ Sinian de façon cruciale. La tension que relève Vera Schwarcz chez les intellectuels chinois de l'ère républicaine entre l'idéal d'une critique régénératrice de la culture, sorte de mouvement des Lumières à la chinoise (qimeng), et l'impératif de sauvetage du pays (jiuguo) ${ }^{67}$ se trouve être au cœur de l'impulsion nouvelle que Fu Sinian donne à son travail. Après le début de l'occupation japonaise, affichant désormais son soutien au Guomindang, l'historien accepte de siéger au Conseil de défense nationale du gouvernement de Chiang Kai-shek. Dans le même temps, il entreprend de réunir ses collègues (Yao Congwu, Jiang Tingfu, Xu Zhongshu, Fang Zhuangyou, Xiao Yishan) autour d'un projet collectif de rédaction d'une histoire générale de la Chine susceptible de répondre aux besoins du présent. L'enjeu est de parvenir à déjouer l'idéologie qui sous-tend l'historiographie impérialiste nipponne des peuples d'Asie orientale. D'après Fu Sinian, les travaux des historiens et idéologues de l'empire japonais n'ont pas d'autre visée que de désolidariser la Mandchourie, la Mongolie et le Tibet du territoire chinois en intégrant l'histoire et les traditions culturelles de ces régions dans l'orbite d'une «civilisation orientale» (tōyō bunmei) alignée sur

chinoise en ce qu'il tend à redéfinir le corpus scripturaire des Classiques hérité du canon confucianiste comme matière proprement historique, et non plus comme ensemble textuel porteur de vérités intemporelles et immuables. Sur le kaozheng, voir Elman (2001). Sur l'instrumentalisation de la tradition du kaozheng par $\mathrm{Hu} \mathrm{Shi}, \mathrm{Gu}$ Jiegang et Fu Sinian, voir Schneider (1971), et Fogel (1995) : 3-21.

67. Voir Schwarcz (1986): 1-12; 286-291. 
une culture japonaise hégémonique ${ }^{68}$. Le projet de Fu Sinian se concrétise par la publication en 1932 d'un Aperçu d'histoire du Nord-Est chinois que l'historien a en réalité écrit seul ${ }^{69}$. Le titre même de l'ouvrage élude l'usage du toponyme de «Mandchourie» (Manzhou) pour réintégrer le territoire dans le cours d'une histoire septentrionale et exclusivement chinoise. Dans ce travail, Fu Sinian analyse, sous un angle à la fois historique et philologique, les mythes et légendes de la Mandchourie ancienne dont il montre les nombreux points communs avec la mythologie des autres régions chinoises à la même époque. Sur le plan anthropologique, il rattache également les coutumes ancestrales des populations du territoire mandchou à celles du peuple han. L'ouvrage fait à l'époque l'objet d'une traduction en anglais due à Li Ji, l'un des pairs et amis de Fu Sinian. Cette version est soumise à la Commission Lytton chargée par la Société des nations de faire la lumière sur l'incident de Mukden de 1931 ayant conduit à la conquête japonaise de la Mandchourie.

Dès sa parution, l'Aperçu d'histoire du Nord-Est chinois de Fu Sinian tombe sous le feu d'une critique virulente. Il est décrié pour son manque de rigueur et d'objectivité par deux jeunes historiens de l'université Zhongyang, Miao Fenglin (1898-1959) et Zheng Hesheng (1901-1989). Connus pour leur penchant conservateur et leur inimitié envers le mouvement pour la «Nouvelle Culture »- dont Fu Sinian avait été l'un des porte-parole dans les années 19101920 -, ces derniers dénoncent dans l'ouvrage un usage très partiel des sources qui repose uniquement sur la Monographie des barbares de l'Est (Dong yi zhuan) et occulte ainsi de nombreux matériaux sur la Mandchourie et ses populations, matériaux pourtant recensés dans les annales chinoises officielles. Les deux historiens ne manquent pas de brocarder un Fu Sinian qui se posait jusqu'alors en parangon d'objectivité et qui, dans la tourmente d'une guerre larvée contre le Japon, semble avoir oublié les préceptes dont il se réclamait en théorie ${ }^{70}$. Force est d'admettre que c'est dans l'urgence de contrer les militaristes japonais et leurs idéologues que Fu Sinian a rédigé à la hâte une telle synthèse. Toutefois, comme le remarque Wang Fan-sen, l'insuffisance des matériaux exploités ne tient pas tant à une volonté délibérée de son auteur de

68. Dans les années 1920 et 1930, l'historien de l'université impériale de Tokyo Yano Jin'ichi (1872-1970) se fait par exemple le porte-voix de cette idéologie qu'il assied dans de nombreux travaux. Voir Yano (1931): 56 sqq.

69. Fu (1932). L'ouvrage, que le PCC refusait jusque dans les années 1980 d'attribuer à $\mathrm{Fu}$ Sinian, historien jugé bien trop réactionnaire en raison de ses liens avec le Guomindang, a fait l'objet d'une réédition récente en Chine continentale qui réhabilite ainsi le nom de son auteur (Shanghai, Shanghai guji chubanshe, 2012). Sur le processus de rédaction du livre et le déni de son auteur par le PCC, voir Hu (1991): 48 sq.

70. Voir Miao (1934): 131 sqq. 
travestir les faits et la réalité qu'à un manque d'expertise de ce dernier. Le fait que Fu Sinian n'ait pas véritablement cherché à répondre aux critiques qui lui étaient adressées à l'époque témoigne qu'il avait bien conscience des limites de ses connaissances en la matière et des lacunes dont souffrait son étude ${ }^{71}$. Mais la mobilisation nationale primait maintenant l'exigence de vérité historique.

\section{Conclusion}

C'est peu dire que la guerre, celle vécue dans le présent du moment comme celle étudiée a posteriori, se confond tout entière dans l'œuvre et la réflexion des intellectuels et historiens chinois de l'ère républicaine. D'un Liang Qichao à un Fu Sinian, en passant par un Jiang Tingfu et un Fan Wenlan, on voit combien elle exacerbe chez eux une conscience aiguë de l'expérience humaine du temps passé et du temps présent. Cette perception acérée de l'histoire qui se fait jour dans un contexte de guerre invite ces derniers à un repositionnement de soi sur le mode de l'action, un repositionnement qui trouve lui-même dans l'histoire, dans ses continuités et ses ruptures, les ressorts de sa propre légitimité et ses directions futures. La portée narrative du travail d'écriture et de réécriture de l'histoire prend ici toute son ampleur. Elle souligne ce qu' on pourrait appeler, pour parler la langue d'un Paul Ricœur, la «condition herméneutique » du sujet historique, c'est-à-dire cet état du sujet se donnant à lui-même «comme lecteur et comme scripteur de sa propre vie» enracinée dans le temps et l'histoire ${ }^{72}$. L'opération historique procède toujours en effet d'un effort interprétatif par lequel, dialoguant avec son temps, le sujet enchâsse dans un récit historique donné son engagement dans l'action politique et sociale. Dans ce récit porteur et vecteur de sens, où convergent les trois temporalités de l'histoire - passé, présent, futur -, le sujet trouve sa raison d'être et de faire. Ainsi, à travers le panégyrique des grands réformateurs de la fin de l'empire dépeints comme autant de lettrés courageux, lucides et conscients des enjeux de leur temps, l'historien Jiang Tingfu justifie jusqu'en 1936 la politique conciliante du Guomindang à l'égard du Japon, en même temps qu'il affirme son attachement au réformisme comme principe d'action politique. Le récit de la révolution d'un Fan Wenlan s'oppose à un tel réformisme et à l'historiographie qui le colporte. Cependant qu'il dénonce le Guomindang et ses historiens pour leurs tendances «réactionnaires», tendances assimilées à l'action des «collaborateurs »

71. Voir Wang (2000): 149-152.

72. Nous empruntons ici la belle formulation de Paul Ricœur, tirée du tome 3 de Temps et récit. Cf. Ricœur (1985): 443. 
d'antan et autres laquais de l'impérialisme occidental et japonais, il donne du grain à moudre à une vision maoïste de l'histoire cherchant elle-même à se départir d'une orthodoxie marxiste qui lui fait entrave en politique. Par son ouvrage de 1932, Fu Sinian entend mobiliser les siens, quitte à s'éloigner d'une objectivité jadis érigée en principe inconditionnel d'étude, autour d'un sentiment indéfectible d'appartenance chinoise qui ne saurait souffrir aucune concession face à l'envahisseur japonais.

Aux modalités narratives et aux enjeux stratégiques de l'histoire que cautionnent les uns et les autres s'ajoute, rappelons-le, l'extrême précarité des conditions matérielles dans lesquelles s'élabore et circule le savoir historien sous la Chine républicaine. Les avatars que l'institutionnalisation des études historiques a connus sous la République en raison du conflit sino-japonais mériteraient de faire l'objet d'une étude à part entière tant le chemin parcouru fut erratique et parsemé d'embuches ${ }^{73}$. Notre regard s'est délibérément détourné de cette dimension institutionnelle de l'historiographie républicaine pour se focaliser sur la réalité historique des représentations de l'expérience humaine que la guerre véhicule, insuffle ou infléchit. L'attention prêtée ici à la littérature historique en tant que pratique culturelle souscrit à l'idée de la possibilité d'une histoire "visant à reconnaître la manière dont les acteurs sociaux donnent sens à leurs pratiques et à leurs discours » ${ }^{74}$. D'où l'intérêt qui nous est apparu de jeter l'éclairage sur l'œuvre de quelques historiens chinois de la République, dans un contexte de guerre qui bouleverse et attaque à la racine les «pratiques» et les «discours».

Le présent article évoquait plus haut l'essai sur l'histoire de Walter Benjamin. Il est loisible de le citer une fois encore pour clore ici la discussion tant son propos fait écho à l'expérience des historiens et intellectuels chinois de l'ère républicaine en lutte avec leur temps. "À chaque époque, écrit le philosophe allemand, il faut chercher à arracher de nouveau la tradition au conformisme qui est sur le point de la subjuguer. [...] Le don d'attiser dans le passé l'étincelle de l'espérance n'appartient qu'à l'historiographe intimement

73. C'est là ce qui ressort du travail de l'historien John Israel sur l'exode des universités de Pékin, Nankai et Qinghua pendant la guerre sino-japonaise. Les trois institutions se réfugient d'abord dans le Hunan, à Changsha, avant de s'exiler dans le Yunnan et de fusionner sous le nom d' «université fédérée du Sud-Ouest» (Xinan lianhe daxue, ou «Lianda»). Voir Israel (1998). Sur la dimension institutionnelle des études historiques en Chine sous l'ère républicaine et leur professionnalisation, voir Moloughney et Zarrow (2011).

74. Chartier (2009): 99-123, et ici: 112. C'est nous qui soulignons. 
persuadé que, si l'ennemi triomphe, même les morts ne seront pas en sûreté. Et cet ennemi n'a pas fini de triompher ${ }^{75}$.»

Le propos laisse songeur en même temps qu'il appelle à la vigilance. Il dit combien il importe de cerner au plus près l'histoire dans les conflits qu'elle donne à voir, même s'il arrive parfois que soit grande la tentation de la laisser s'en retourner, libre de toute appréhension humaine, sur les chemins de son équivoque; de guerre lasse.

\section{Bibliographie}

ARENDT Hannah (1972). «La brèche entre le passé et le futur ». In La Crise de la culture. Paris, Gallimard, «Folio Essais ».

BEAUPRÉ Nicolas (2006). Écrire en guerre, écrire la guerre. France, Allemagne 19141920. Paris, CNRS Éditions.

BECKER Annette (1998). Journaux de combattants et de civils de la France du Nord dans la Grande Guerre. Villeneuve-d'Ascq, Presses universitaires du Septentrion.

BECKER Annette (2012). Oubliés de la Grande Guerre. Humanitaire et culture de guerre, 1914-1918. Paris, Pluriel.

Benjamin Walter (2000). «Sur le concept d'histoire». In Euvres, vol. 3. Paris, Gallimard.

Bergère Marie-Claire, BiAnco Lucien et Domes Jürgen (dir.) (1989). La Chine au Xx siècle. D’une révolution à l'autre (1895-1949). Paris, Fayard.

Black Cyril E. (1966). The Dynamics of Modernization. A Study in Comparative History. New York, Harper.

BrooK Timothy (1990). "Capitalism and the Writing of Modern History in China». In Gregory Blue et Timothy Brook (dir.), China and Historical Capitalism. Genealogies of Sinological Knowledge. Cambridge, Cambridge University Press.

CHARTIER Roger (2009). «L'histoire entre récit et connaissance». In Au bord de la falaise, l'histoire entre certitudes et inquiétudes. Paris, Albin Michel.

Chen Hsi-yuan (2006). «Last Chapter Unfinished: The Making of the Official Qing History and the Crisis of Traditional Historiography ». Historiography East and West, $\mathrm{n}^{\circ} 2$ : 173-204.

Cheng Anne (2003). «Modernité et invention de la tradition chez les intellectuels chinois du Xx $x^{\mathrm{e}}$ siècle». In Yves Michaud (dir.), La Chine aujourd'hui. Paris, Odile Jacob, «Université de tous les savoirs».

Chesneaux Jean (1976). Le Mouvement paysan chinois, 1840-1949. Paris, Seuil.

Chevrier Yves (1984). «Chine, "fin de règne" du lettré? Politique et culture à l'époque de l'occidentalisation». Extrême-Orient, Extrême-Occident, n ${ }^{\circ}$ : : 81-139.

75. Benjamin (2000): 431. 
CHow Tse-tsung (1960). The May Fourth Movement. Intellectual Revolution in Modern China. Cambridge (Mass.), Harvard University Press.

Delacroix Christian, Dosse François et Garcia Patrick (2007). Les Courants historiques en France, $\mathrm{XIX}-\mathrm{XX}^{e}$ siècle. Paris, Gallimard, «Folio Histoire».

Ding Wenjiang 丁文江 et ZHAO Fengtian 趙豐田 (1983). Liang Qichao nianpu changbian 梁啟超年譜長編 (Biographie chronologique de Liang Qichao). Shanghai, Shanghai renmin chubanshe.

DIRLIK Arif (2001). Revolution and History. The Origins of Marxist Historiography in China, 1919-1937. Berkeley, University of California Press.

Elman Benjamin A. (2001). From Philosophy to Philology. Intellectual and Social Aspects of Change in Late imperial China. Los Angeles, University of California, «UCLA Asian Pacific Monograph Series».

FAN Wenlan 范文瀾 (1956). Nü gemingjia Qiu Jin 女革命家秋瑾 (Qiu Jin, femme révolutionnaire). Zhongguo funü, $\mathrm{n}^{\circ} 8: 20-22$.

FAN Wenlan 范文瀾 (1989). Zhongguo tongshi jianbian 中國通史簡編 (Précis d'histoire générale de Chine). Shanghai, Shanghai shuju.

FAN Wenlan 范文瀾 (1992). Zhongguo jindaishi 中國近代史 (Histoire de la Chine moderne). Shanghai, Shanghai shudian, «Minguo congshu, IV», vol. 78.

Fogel Joshua A. (1995). «On the "Rediscovery" of the Chinese Past: Cui Shu and Related Cases». In Joshua A. Fogel (dir.), Cultural Dimension of Sino-Japanese Relations. Essays on the Nineteenth and Twentieth Centuries. Armonk, M. E. Sharpe.

Fu Sinian 傅斯年 (1932). Dongbei shigang 東北史綱 (Aperçu d'histoire du Nord-Est chinois). Pékin, Institut d'histoire et de philologie de l'Academia Sinica.

Goldman Merle et Lee Leo Ou-fan (dir.) (2002). An Intellectual History of Modern China. Cambridge, Cambridge University Press.

Goody Jack (2006). The Theft of History. Cambridge, Cambridge University Press.

GraY J. (1961). «Historical Writing in Twentieth-Century China: Notes on its Background and Development». In William G. Beasley et Edwin G. Pulleyblank (dir.), Historians of China and Japan. Londres, Oxford University Press.

Grieder Jerome B. (1970). Hu Shih and the Chinese Renaissance. Liberalism in the Chinese Revolution, 1917-1937. Cambridge (Mass.), Harvard University Press.

Hartog François (2013). Croire en l'histoire. Paris, Flammarion.

Hu Houxuan 胡厚宣 (1991). Dongbei shigang (di yi juan) de zuozhe shi Fu Sinian 東北史綱 (第一卷) 的作者是傅斯年 (Fu Sinian, auteur du premier volume de l'Aperçu d'histoire du Nord-Est chinois). Shixueshi yanjiu, nº 3: 48-49.

Huang Kewu 黃克武 (2006). Yi ge bei fangqi de xuanze. Liang Qichao tiaoshi sixiang zhi yanjiu 一個被放育的選擇 : 梁啟超調適思想之研究 (Une voie oubliée. Recherche sur la pensée adaptative de Liang Qichao). Taipei, Institut d'histoire moderne de l'Academia Sinica.

Huang Philip C. (1972). Liang Ch'i-ch'ao and Modern Chinese Liberalism. Seattle, University of Washington Press. 


\section{Damien Morier-Genoud}

Hummel Arthur W. (trad.) (1931). Autobiography of a Chinese Historian. Being the Preface to a Symposium on Ancient Chinese History (Ku Shih Pien). Leyden, Late E. J. Brill Ltd.

IsRael John (1998). Lianda. A Chinese University in War and Revolution. Stanford (Ca.), Stanford University Press.

JIANG Tingfu 蔣廷楼 (1971a). Qishan yu yapian zhanzheng 琦善與鴉片戰爭 (Qishan et la [première] guerre de l’Opium). In Jiang Tingfu xuanji 蔣廷䊀選集 (Euvres choisies de Jiang Tingfu), vol. 1. Taipei, Zhuanji wenxue chubanshe.

JIANG Tingfu 蔣廷韨 (1971b). Wei shi de jiangtu shi women de chulu 未失的疆土是我 們的出路 (Le territoire non occupé est notre échappatoire). In Jiang Tingfu xuanji 蔣廷䟣選集 (CEuvres choisies de Jiang Tingfu), vol. 2. Taipei, Zhuanji wenxue chubanshe.

JIANG Tingfu 蔣廷䊋 (1989a). Jindai Zhongguo waijiaoshi ziliao jiyao 近代中國外交 史資料輯要 (Synthèse des matériaux sur l'histoire de la politique étrangère de la Chine moderne). Shanghai, Shanghai shudian, «Minguo congshu, II », vol. 27.

JIANG Tingfu 蔣廷䊋 (1989b). Zhongguo jindaishi 中國近代史 (Histoire de la Chine moderne). Shanghai, Shanghai shudian, «Minguo congshu, II , vol. 75.

JIANG Tingfu 蔣廷馛 (2003). Jiang Tingfu huiyilu 蔣廷馛回憶錄 (Mémoires de Jiang Tingfu). Changsha, Yuelu shushe.

Koselleck Reinhart (1990). Le Futur passé. Paris, École des hautes études en sciences sociales.

LeVenson Joseph R. (1959). Liang Ch'i-ch'ao and the Mind of Modern China. Londres, Thames and Hudson.

Leys Simon (1998). «Ces experts qui nous expliquent la Chine». In Essais sur la Chine. Paris, Robert Laffont, «Bouquin».

Li Huaiyin (2013). Reinventing Modern China. Imagination and Authenticity in Chinese Historical Writing. Honolulu, University of Hawai'i Press.

Liang Qichao 梁啟超 (1936a). Ziyou shu 自由書 (Livre des écrits de liberté). In Yinbingshi heji zhuanji 飲冰室合集專集 (Morceaux choisis des Euvres du Studio du buveur de glace), vol. 2. Shanghai, Zhonghua shuju.

LIANG Qichao 梁啟超 (1936b). Ouyou xinyinglu jielu 歐遊心影錄節錄 (Compendium des Impressions de voyage en Europe). In Yinbingshi heji zhuanji 飲冰室合集 專集 (Morceaux choisis des Euvres du Studio du buveur de glace), vol. 23. Shanghai, Zhonghua shuju.

LIANG Qichao 梁啟超 (1936c). Ouzhou zhanyi shilun 歐洲戰役史論 (Essai d'histoire sur la Grande Guerre en Europe). In Yinbingshi heji zhuanji 飲冰室合集專集 (Morceaux choisis des Euvres du Studio du buveur de glace), vol. 30. Shanghai, Zhonghua shuju.

LiANG Qichao 梁啟超 (1936d). Sanshi zishu 三十自述 (Autobiographie au seuil de la trentaine). In Yinbingshi heji wenji 飲冰室合集文集 (Essais choisis des Euvres du Studio du buveur de glace), vol. 11. Shanghai, Zhonghua shuju.

LiANG Qichao 梁啟超 (1980a). Xin shixue 新史學 (Renouveler l'historiographie). In Lin Yi 林毅 (éd.), Liang Qichao shixue lunzhu san zhong 梁啟超史學論著三種 (Trois essais de Liang Qichao sur l'historiographie). Hongkong, Sanlian shudian. 
LIANG Qichao 梁啟超 (1980b). Zhongguo lishi yanjiufa 中國歷史研究法 (Méthodologie de la recherche pour l'histoire de Chine). In Lin Yi 林毅 (éd.), Liang Qichao shixue lunzhu san zhong 梁啟超史學論著三種 (Trois essais de Liang Qichao sur l'historiographie). Hongkong, Sanlian shudian.

LIANG Qichao 梁啟超 (1989). Zhongguo lishi yanjiufa bubian 中國歷史研究法補 編 (Appendice de la Méthodologie de la recherche pour l'histoire de Chine). Shanghai, Shanghai shudian, «Minguo congshu, I», vol. 73.

Lin Yu-sheng (1979). The Crisis of Chinese Consciousness. Radical Antitraditionalism in the May Fourth Era. Madison, University of Wisconsin Press.

LiU Yao 劉耀 (1983). Jiang Tingfu de wenhua shiguan yu Zhongguo jindaishi 蔣廷戕 的文化史觀與中國近代史 (La conception historique de la culture et l'histoire de la Chine moderne selon Jiang Tingfu). Renwen zazhi, $\mathrm{n}^{\circ}$ 6: 87-91.

Luo Rongqu 羅榮渠 (1993). Xiandaihua xinlun. Shijie yu Zhongguo de xiandaihua jincheng 現代化新論：世界與中國的現代化進程 (Une théorie nouvelle de la modernisation. Le processus modernisateur de par le monde et la Chine). Pékin, Beijing daxue chubanshe.

MARrou Henri-Irénée (1968). Théologie de l'histoire. Paris, Seuil.

Miao Fenglin 繆鳳林 (1934). Ping Fu Sinian jun Dongbei shigang juanshou 評傅斯 年君東北史剛卷首 (À propos du volume introductif de l'Aperçu d'histoire du Nord-Est chinois de M. Fu Sinian). Wenyi congkan, vol. 2, n 2 : 131-163.

Moloughney Brian et Zarrow Peter (dir.) (2011). Transforming History. The Making of a Modern Academic Discipline in Twentieth-Century China. Hongkong, The Chinese University Press.

Morier-Genoud Damien (2014). «Critique et méthodologie de l'histoire dans la Chine républicaine. Divergences de vues entre Fu Sinian et Qian $\mathrm{Mu}$ ». In Nathalie Kouamé (dir.), Historiographies d'ailleurs. Comment écrit-on l'histoire en dehors du monde occidental?, Paris, Karthala.

Riceur Paul (1985). Temps et récit. Paris, Seuil.

Romilly Jacqueline de (2005). L'Invention de l'histoire politique chez Thucydide. Paris, Éditions rue d'Ulm.

Roux Alain (2004). La Chine au xx siècle. Paris, Armand Colin, «Campus Histoire». Rozman Gilbert (dir.) (1981). The Modernization of China. New York, The Free Press.

SCHILLER J. C. Friedrich von (1872). «Qu'est-ce que l'histoire universelle et pourquoi l'étudie-t-on?» In Ad. Regnier (dir.), Euvres historiques de Schiller, vol. 5. Paris, Librairie Hachette.

SCHNEIDER Laurence A. (1971). Ku Chieh-kang and China's New History. Nationalism and the Quest for Alternative Traditions. Berkeley, University of California Press.

Schwarcz Vera (1986). The Chinese Enlightenment. Intellectuals and the Legacy of the May Fourth Movement of 1919. Berkeley, University of California Press.

TANG Xiaobing (1996). Global Space and the Nationalist Discourse of Modernity. The Historical Thinking of Liang Qichao. Stanford (Calif.), Stanford University Press.

TraVERSO Enzo (2011). L'Histoire comme champ de bataille. Interpréter les violences $d u \times X^{e}$ siècle. Paris, La Découverte. 


\section{Damien Morier-Genoud}

Valéry Paul (1957). «La Crise de l'esprit». In CEuvres, vol. 1. Paris, Gallimard, «Bibliothèque de la Pléiade».

WANG Fan-sen [Wang Fansen] 王汎森 (1987). Gushi bian yundong de xingqi 古史辨 運動的興起 (La naissance du mouvement des Discussions critiques sur l'histoire ancienne). Taipei, Yunchen wenhua gongsi.

WANG Fan-sen (2000). Fu Ssu-nien. A Life in Chinese History and Politics. Cambridge, Cambridge University Press.

WANG Q. Edward (2001). Inventing China Through History. The May Fourth Approach to Historiography. Albany, State University of New York Press.

Weigelin-Schwierdrzik Susanne (2007). «Back to the Past: Chinese Intellectuals in Search of Historical Legitimacy (1957-1965)». In Mechthild Leutner (dir.), Rethinking China in the 1950s. Münster: LIT, «Chinese History and Society, vol. $31 »$.

Wu Huaiqi 吳懷祺 (2002). Zhongguo shixue sixiang tongshi 中國史學思想通史 (Histoire générale de la pensée historiographique en Chine). Hefei, Huangshan shushe.

YANO Jin'ichi 矢野仁一 (1931). Man-Mō-Zō wa Shina honrai no ryōdo ni arazu ron 滿 蒙藏は支那本來の領土に非る論 (Sur la non-appartenance de la Mandchourie, de la Mongolie et du Tibet au territoire chinois originel). Gaikō jihō, vol. 35, $\mathrm{n}^{\circ} 412$ : 56-71.

YU Ying-shih (1982). «The Study of Chinese History: Retrospect and Prospect». In George Kao (dir.), The Translation of Things Past. Chinese History and Historiography. Hongkong, Chinese University Press.

Yu Ying-shih (1994). «Changing Conceptions of National History in Twentieth-Century China». In Erik Lönnroth, Karl Molin et Ragnar Björk (dir.), Conceptions of National History. Proceedings of Nobel Symposium 78. Berlin, Walter de Gruyter.

\section{Glossaire}

ban fengjian de 半封建的

ban zhimin de 半殖民的

beifa 北伐

Cai Hesen 蔡和森

chang gaodiao 唱高調

Chen Gonglu 陳恭錄

Chiang Kai-shek (Jiang Jieshi) 蔣介石

Chuanbi 穿鼻

dadan jiashe, xiaoxin qiuzheng 大膽假設, 小心求證

Dai Jitao 戴季陶

Daxue 大學

Ding Wenjiang 丁文江 
Dong yi zhuan 東夷傳

Duli pinglun 獨立評論

ershisi xing zhi jiapu 二十四姓之家譜

Fan Wenlan 范文瀾

Fang Zhuangyou 方壯猷

fangshou maiguo 放手賣國

fangzhi 方志

Feng Guifen 馮桂芬

$\mathrm{Fu}$ Sinian 傅斯年

fu yi 撫夷

Gong Zizhen 竟自珍

gongche shangshu 公車上書

Gu Jiegang 顧頡剛

guojia yingxiong 國家英雄

guoxue 國學

Gushi bian 古史辩

Hu Hanmin 胡漢民

$\mathrm{Hu}$ Shi 胡適

Huang Kan 黃㑆

Jiang Tingfu 蔣廷韨

jiao yi 彩夷

Jigongshan 雞公山

jiuguo 救國

Kang Youwei 康有爲

Li Da 李達

Li Dazhao 李大釗

Li Dingsheng 李鼎聲

Li Huaiyin 李懷印

$\mathrm{Li} \mathrm{Ji}$ 李濟

Liang Qichao 梁啟超

Lin Zexu 林則徐

Liu Zhiji 劉知幾

Manzhou 滿洲

Mao Zedong 毛澤東

Miao Fenglin 繆鳳林

Muzhang'a 穆彰阿

Qingshi guan 清史館

Qishan 琦善

Qiu Jin 秋瑾

Qu Qiubai 篗秋白 


\section{Damien Morier-Genoud}

renlei lishi de zhuanlie 人類歷史的轉捩

shiguan xuepai 史觀學派

shijie geming 史界革命

shiliao xuepai 史料學派

Sima Guang 司馬光

Sun Jianai 孫家舀

Sun Yat-sen (Sun Zhongshan) 孫中山

Taiping 太平

tōyō bunmei 東洋文明

tudi geming 土地革命

Wang Fan-sen 王汎森

Wanguo gongbao 萬國公報

Wei Yuan 魏源

Wenxin diaolong 文心彫龍

Xiao Yishan 蕭一山

xin minzhuzhuyi geming 新民主主義革命

$x$ in 新

Xinhui 新會

Xinmin congbao 新民叢報

Xinmin shuo 新民說

xinmin 新民

Xu Jiyu 徐繼蕃

Xu Zhongshu 徐中舒

Yano Jinichi 矢野仁一

Yao Congwu 姚從吾

Yu Ying-shih 余英時

Yuan Shikai 袁世凱

Zhang Wentian 張聞天

Zhang Xuecheng 章學誠

Zhao Yi 趙翼

Zhedong 浙東

Zheng Hesheng 鄭鶴聲

zhengshi 正史

zhi you chaoting er bu zhi you guojia 知有朝廷而不知有國家

zhi you chenji er bu zhi you jinwu 知有陳跡而不知有今務

zhi you geren er bu zhi you qunti 知有個人而不知有珜體

zhi you shishi er bu zhi you lixiang 知有事實而不知有理想

Zhongguo tongshi jianbian 中國通史簡編

Zhongwai jiwen 中外紀聞

ziqiang 自強 